JOURNAL OF LATEX CLASS FILES, VOL. 6, NO. 1, JANUARY 2007

\title{
Universal Wyner-Ziv Coding for Distortion Constrained General Side-Information
}

\author{
Shun Watanabe Member, IEEE and Shigeaki Kuzuoka Member, IEEE
}

\begin{abstract}
We investigate the Wyner-Ziv coding in which the statistics of the principal source is known but the statistics of the channel generating the side-information is unknown except that it is in a certain class. The class consists of channels such that the distortion between the principal source and the side-information is smaller than a threshold, but channels may be neither stationary nor ergodic. In this situation, we define a new rate-distortion function as the minimum rate such that there exists a Wyner-Ziv code that is universal for every channel in the class. Then, we show an upper bound and a lower bound on the rate-distortion function, and derive a matching condition such that the upper and lower bounds coincide. The relation between the new rate-distortion function and the rate-distortion function of the Heegard-Berger problem is also discussed.
\end{abstract}

\section{Index Terms}

Average Distortion, Heegard-Berger Problem, Maximum Distortion, Universal Coding, Wyner-Ziv Problem

\section{INTRODUCTION}

In the seminal paper [1], Wyner and Ziv characterized the rate-distortion function of the lossy source coding with side-information at the decoder (See Fig. 11). In this paper, we consider a universal coding of this problem where the statistics of the principal source is known but the channel from the principal source to the side-information is unknown except that it is in a certain class.

To motivate the problem setting investigated in this paper, let us consider the following practical situation first. Suppose that the decoder already has a lossy compressed version of the principal source, and want to get a refined one. The encoder does not know how the previously transmitted lossy version is encoded, but knows that the quality of the lossy version is guaranteed to be above a certain level. What is the minimum additional rate that must be transmitted by the encoder so that the quality of the refined version is above a required level?

The first author is with the Department of Information Science and Intelligent Systems, University of Tokushima, 2-1, Minami-josanjima, Tokushima, 770-8506, Japan, e-mail:shun-wata@is.tokushima-u.ac.jp.

The second author is with the Department of Computer and Communication Sciences, Wakayama University, Wakayama, 640-8510, Japan, e-mail:kuzuoka@ieee.org.

Manuscript received ; revised 
The above mentioned situation can be modeled as follows. The principal source $X^{n}$ is a known i.i.d. source, and the side-information $Y^{n}$ is generated from $X^{n}$ through a channel $W^{n}$. The statistical property of the channel is unknown, but the distortion caused by the channel is smaller than a certain level $E$ for a prescribed distortion measure. We assume that the distortion measure is additive, but the channel may be neither stationary nor ergodic. We consider the maximum distortion constraint and the average distortion constraint for the channel. Since we allow non-ergodic channel, the class of channels constrained by the maximum distortion and that constrained by the average distortion are different. In this problem formulation, we are interested in the minimum rate $R_{m}(D \mid E)$ and $R_{a}(D \mid E)$ such that the reproduction with distortion level $D$ is possible at the decoder for any channel in the classes of channels satisfying the distortion level $E$ with the maximum distortion constraint and the average distortion constrain respectively. In other word, we are interested in the minimum rate such that the universal coding is possible for each class.

For the maximum distortion constrained class, we show an upper bound and a lower bound on $R_{m}(D \mid E)$. We also derive a matching condition such that the upper and the lower bounds coincide. Especially, for the binary Hamming example, we show that the matching condition is satisfied, and thus $R_{m}(D \mid E)$ is completely characterized.

For the average distortion constrained class, we show an upper bound and a lower bound on $R_{a}(D \mid E)$. For the case with $D=0$, i.e., the loss less reproduction case, we show that the upper and lower bounds coincide and thus $R_{a}(0 \mid E)$ is completely characterized. Surprisingly, $R_{a}(0 \mid E)=H(X)$, i.e., the side-information is completely useless, for any $E>0$.

Some remarks on related literatures are in order.

For lossless source coding with side-information, i.e., the Slepian-Wolf network [2], the existence of universal code was first shown by Csiszár and Körner [3] (existence of linear universal code was also shown by Csiszár [4]). After that, the universal codings for the Slepian-Wolf network or other related lossless multi-terminal networks were studied by several researchers [5], [6], [7].

For lossy source coding with side-information, i.e., the Wyner-Ziv network, the universal coding problem was investigated by Merhav and Ziv [8], Jalali et. al. [9], and Reani and Merhav [10]. It should be noted that the universal codes proposed in these literatures are universal for the statistics of the principal source but not for the channel generating the side-information, i.e., the statistics of the channel is known at the encoder. Under the same condition, i.e., known channel, it is also known that the universal code can be constructed for the network with several decoders [11].

The universal Wyner-Ziv coding is also related to the Heeger-Berger problem [12], in which there are several decoders that have their own side-information. The Heeger-Berger problem has not been solved in general, and it has only been solved under the condition that there is a degraded partial order between the channels generating the side-information [13], [14], [15] except some special cases [16], [17]. It should be noted that there is no degraded partial order among the channel class considered in this paper. Thus, the authors believe that the result in this paper also shed some light on the unsolved Heeger-Berger problem.

Our problem setting can be also viewed as a kind of the successive refinement coding [18], [19]. The successive 


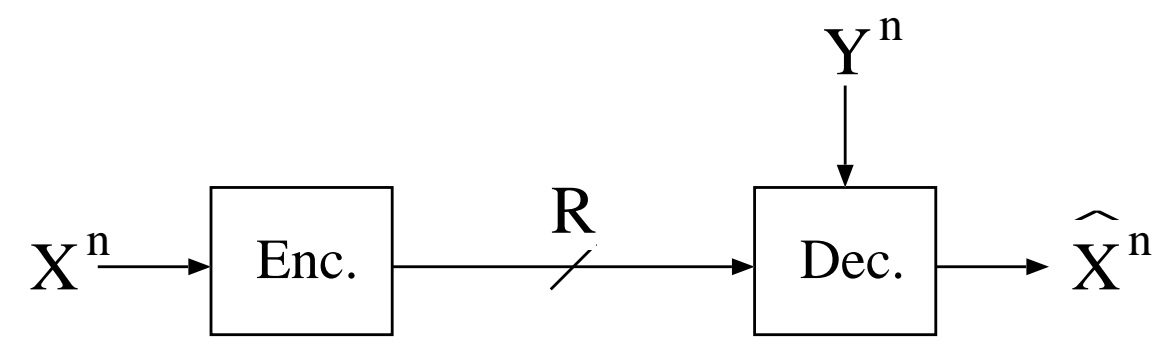

Fig. 1. The Wyner-Ziv coding system.

refinement coding consists of two layers of the encodings. If the method used by the first layer encoder is not known to the second layer encoder, this is exactly the situation of our problem setting.

Although the universal coding for distortion constrained class of channels is unfamiliar and new in the source coding scenario, this kind of channel is quite natural when the channel is cased by an adversary such as in the data hiding scenario. Indeed, this kind of channel class is commonly used in the information theoretical analysis of the data hiding [20], [21], [22].

There are some technical differences between the data hiding problem and our problem. First, in the data hiding problem, the channel output is only used for the decoding of the encoded message. On the other hand, in our problem, the side-information is not only used for the decoding of the encoded source, but also for the estimation at the decoder. This makes the problem difficult, and causes a gap between the upper bound and the lower bound derived in this paper. Second, in the data hiding problem for the average distortion constrained class of channels, it was shown that the achievable transmission rate is 0 , i.e., the channel is completely useless [21]. On the other hand, in our problem for the average distortion constrained class of channels, the side-information is useless for bin coding, but it can be used for the estimation at the decoder. Thus, $R_{a}(D \mid E)$ can be strictly smaller than the rate-distortion function $R(D)$ without any side-information for $D>0$, though $R_{a}(0 \mid E)=H(X)$.

The rest of this paper is organized as follows. In Section $\llbracket$ we introduce notations and the formal definition of the problem. In Section III, we state our main theorems, and show a representative example, i.e., the binary Hamming example. In Sections IV] and $\mathrm{V}$, we present proofs of the main theorems.

\section{PREliminaries}

\section{A. Notations}

Henceforth, we adopt the following notation conventions. Random variables will be denoted by capital letters such as $X$, while their realizations will be denoted by respective lower case letters such as $x$. A random vector of length $n$ is denoted by $X^{n}=\left(X_{1}, \ldots, X_{n}\right)$, while its realization is denoted by $x^{n}=\left(x_{1}, \ldots, x_{n}\right)$. The alphabet of a random variable is denoted by a calligraphic letter such as $\mathcal{X}$, and its $n$-fold Cartesian product is denoted by $\mathcal{X}^{n}$. The probability distribution of random variable $X$ is denoted by $P_{X}$, and its $n$-fold i.i.d. extension is denoted by $P_{X}^{n}$. For a given channel $W$, its $n$-fold i.i.d. extension is denoted by $W^{\times n}$, while $W^{n}$ indicates a channel that is not 
necessarily i.i.d.. The set of all probability distribution on $\mathcal{X}$ is denoted by $\mathcal{P}(\mathcal{X})$. The set of all channel from $\mathcal{X}$ to $\mathcal{Y}$ is denoted by $\mathcal{P}(\mathcal{Y} \mid \mathcal{X})$. The indicator function is denoted by $\mathbf{1}[\cdot]$. The entropy and the mutual information is denoted in a standard notation such as $H(X)$ or $I(X ; Y)$. For a input distribution $P$ of a channel $W$, we sometimes use the notation $I(P, W)$ to designate the mutual information $I(X ; Y)$, where the joint distribution of $(X, Y)$ is $P(x) W(y \mid x)$. The variational distance between two distributions $P$ and $Q$ is denoted by $\|P-Q\|$. In the proofs of our main theorems, we extensively use the type and typicality, which are summarized in Appendix $\mathrm{C}$

\section{B. Problem Formulation}

Let $\boldsymbol{X}=\left\{X^{n}\right\}_{n=1}^{\infty}$ be an i.i.d. source. Let

$$
e_{n}\left(x^{n}, y^{n}\right):=\frac{1}{n} \sum_{t=1}^{n} e\left(x_{t}, y_{t}\right)
$$

be an additive distortion measure for side information. As a natural assumption, we assume that there exists $y$ such that $e(x, y)=0$ for each $x$. We also assume that the distortion is bounded, i.e., $e(x, y) \leq e_{\max }<\infty$ for every $(x, y)$. For a given distortion $E \geq 0$, we consider the following maximum distortion constraint on the side-information

$$
\begin{aligned}
\mathcal{W}_{m}(E):= & \left\{\boldsymbol{W}=\left\{W^{n}\right\}_{n=1}^{\infty}: \forall \delta>0 \exists n_{0}(\delta)\right. \text { s.t. } \\
& \left.\operatorname{Pr}\left\{e_{n}\left(X^{n}, Y^{n}\right)>E\right\} \leq \delta \forall n \geq n_{0}(\delta)\right\},
\end{aligned}
$$

where $Y^{n}$ is the output of channel $W^{n}$ with input $X^{n}$. It should be noted that $n_{0}(\delta)$ depends on $\delta$ but not on $\boldsymbol{W}$. We also consider the average distortion constraint

$$
\begin{aligned}
& \mathcal{W}_{a}(E) \\
& \quad:=\quad\left\{\boldsymbol{W}=\left\{W^{n}\right\}_{n=1}^{\infty}: e_{n}\left(P_{X^{n}}, W^{n}\right) \leq E \forall n \geq 1\right\}
\end{aligned}
$$

where

$$
\begin{aligned}
e_{n}\left(P_{X^{n}}, W^{n}\right) & :=\mathbb{E}\left[e_{n}\left(X^{n}, Y^{n}\right)\right] \\
& =\sum_{x^{n}, y^{n}} P_{X}^{n}\left(x^{n}\right) W^{n}\left(y^{n} \mid x^{n}\right) e_{n}\left(x^{n}, y^{n}\right) .
\end{aligned}
$$

As it will be clarified later, the maximum distortion constraint and the average distortion constraint are completely different.

Let $\hat{\mathcal{X}}$ be the reproduction alphabet. Then, let

$$
d_{n}\left(x^{n}, \hat{x}^{n}\right):=\frac{1}{n} \sum_{t=1}^{n} d\left(x_{t}, \hat{x}_{t}\right)
$$

be an additive distortion measure for reproduction. We assume $d(x, \hat{x}) \leq d_{\max }<\infty$ for every $(x, \hat{x})$. We also assume that for each $x$ there exists $\hat{x}$ such that $d(x, \hat{x})=0$.

We consider (possibly stochastic) encoder

$$
\varphi_{n}: \mathcal{X}^{n} \rightarrow \mathcal{M}_{n}
$$


and decoder

$$
\psi_{n}: \mathcal{M}_{n} \times \mathcal{Y}^{n} \rightarrow \hat{\mathcal{X}}^{n} .
$$

Definition 1: For any $\varepsilon>0$, if there exists $n_{0}(\varepsilon)$ and a sequence of codes $\left\{\left(\varphi_{n}, \psi_{n}\right)\right\}_{n=1}^{\infty}$ such that

$$
\frac{1}{n} \log \left|\mathcal{M}_{n}\right| \leq R+\varepsilon
$$

and

$$
\mathbb{E}\left[d_{n}\left(X^{n}, \psi_{n}\left(\varphi_{n}\left(X^{n}\right), Y^{n}\right)\right)\right] \leq D+\varepsilon
$$

for every $\boldsymbol{W} \in \mathcal{W}_{m}(E)$ and $n \geq n_{0}(\varepsilon)$, then we define the rate $R$ to be achievable. We also define the rate distortion function

$$
R_{m}(D \mid E):=\inf \{R: R \text { is achievable }\} .
$$

We also define $R_{a}(D \mid E)$ by replacing $\mathcal{W}_{m}(E)$ with $\mathcal{W}_{a}(E)$.

From the problem formulation, we can prove the following relation between $R_{m}(D \mid E)$ and $R_{a}(D \mid E)$, which will be proved in Appendix $\mathrm{A}$

Proposition 2: We have

$$
R_{a}(D \mid E) \geq \lim _{\epsilon \downarrow 0} R_{m}(D \mid E-\epsilon) .
$$

Remark 3: As we can find from the proof of Theorem 6, the theorem holds even if the average distortion requirement in (5) is replaced by the maximum distortion requirement

$$
\operatorname{Pr}\left\{d_{n}\left(X^{n}, \psi_{n}\left(\phi_{n}\left(X^{n}\right), Y^{n}\right)\right)>D+\varepsilon\right\} \leq \varepsilon .
$$

However, Theorem 10 does not hold if (5) is replaced by (6).

Let $R_{W Z}(D \mid W)$ be the rate distortion function of the ordinary Wyner-Ziv problem in which the principal source is $X$ and the side-information $Y$ is the output of the channel $W \in \mathcal{P}(\mathcal{Y} \mid \mathcal{X})$.

The rate distortion function $R_{m}(D \mid E)$ (or $R_{a}(D \mid E)$ ) means that if $R>R_{m}(D \mid E)$ there exists a universal code that works well for every $\boldsymbol{W} \in \mathcal{W}_{m}(E)$ (or $\boldsymbol{W} \in \mathcal{W}_{a}(E)$ ). It should be noted that this definition of universality is different from the ordinary definition of the universality. Let

$$
\mathcal{W}_{W Z}(R, D):=\left\{W \in \mathcal{P}(\mathcal{Y} \mid \mathcal{X}): R_{W Z}(D \mid W) \leq R\right\}
$$

In the ordinary definition of the universality, we require that there exists a code that works well for every $W \in$ $\mathcal{W}_{W Z}(R, D)$. This requirement seems much more severe than the requirement of $R_{m}(D \mid E)$ (or $R_{a}(D \mid E)$ ), which will be discussed in more detail in Section 


\section{Heegard-Berger Problem}

For later use, we review the problem formulation of the Heegard-Berger (HB) problem [12] in this section. We restrict our attention to the case with two decoders (see Fig. 2). Furthermore, we restrict our attention to the case such that the alphabets of the side-information, the reproduction alphabets, and the distortion measures for both the decoders are common, which are denoted by $\mathcal{Y}, \hat{\mathcal{X}}$, and $d(\cdot, \cdot)$ respectively.

Let us consider the HB coding for i.i.d. joint source $\left(X, Y_{1}, Y_{2}\right)$. The HB code consists of one encoder

$$
\varphi_{n}^{H B}: \mathcal{X}^{n} \rightarrow \mathcal{M}_{n}
$$

and two decoders

$$
\begin{aligned}
& \psi_{n}^{H B 1}: \mathcal{M}_{n} \times \mathcal{Y}^{n} \rightarrow \hat{\mathcal{X}}^{n}, \\
& \psi_{n}^{H B 2}: \mathcal{M}_{n} \times \mathcal{Y}^{n} \rightarrow \hat{\mathcal{X}}^{n} .
\end{aligned}
$$

For a pair $\left(D_{1}, D_{2}\right)$ of distortions, a rate $R$ is defined to be $\left(D_{1}, D_{2}\right)$-achievable if, for any $\varepsilon>0$, there exists a sequence of HB code $\left\{\left(\varphi_{n}^{H B}, \psi_{n}^{H B 1}, \psi_{n}^{H B 2}\right)\right\}_{n=1}^{\infty}$ such that

$$
\begin{aligned}
\frac{1}{n} \log \left|\mathcal{M}_{n}\right| & \leq R+\varepsilon \\
\mathbb{E}\left[d_{n}\left(X^{n}, \hat{X}_{i}^{n}\right)\right] & \leq D_{i}+\varepsilon \quad i=1,2,
\end{aligned}
$$

for sufficiently large $n$, where $\hat{X}_{i}^{n}=\psi_{n}^{H B i}\left(\varphi_{n}\left(X^{n}\right), Y_{i}^{n}\right)$ and $d_{n}$ is defined in (3). Then, the HB rate-distortion function $R_{H B}\left(D_{1}, D_{2} \mid X, Y_{1}, Y_{2}\right)$ for $\left(X, Y_{1}, Y_{2}\right)$ is defined as the infimum of $\left(D_{1}, D_{2}\right)$-achievable rate $R$.

Fix an i.i.d. source $P_{X}$. Then two side-information channel $W_{1}: \mathcal{X} \rightarrow \mathcal{Y}$ and $W_{2}: \mathcal{X} \rightarrow \mathcal{Y}$ define an i.i.d. joint source $\left(X, Y_{1}, Y_{2}\right)$ whose joint distribution $P_{X Y_{1} Y_{2}}$ is given by $P_{X Y_{1} Y_{2}}\left(x, y_{1}, y_{2}\right)=P_{X}(x) W_{1}\left(y_{1} \mid x\right) W_{2}\left(y_{2} \mid x\right)$, where $x \in \mathcal{X}$ and $y_{1}, y_{2} \in \mathcal{Y}$. In the following, we denote by $R_{H B}\left(D_{1}, D_{2} \mid W_{1}, W_{2}\right)$ the HB rate-distortion function $R_{H B}\left(D_{1}, D_{2} \mid X, Y_{1}, Y_{2}\right)$ for $\left(X, Y_{1}, Y_{2}\right)$ defined by $W_{1}$ and $W_{2}$.

Unfortunately, finding a single-letter expression for $R_{H B}\left(D_{1}, D_{2} \mid W_{1}, W_{2}\right)$ has been a long-standing open problem. So, we consider a special case. Let

$$
E_{*}:=\min _{y \in \mathcal{Y}} \sum_{x \in \mathcal{X}} P_{X}(x) e(x, y)
$$

and $y_{*} \in \mathcal{Y}$ be a symbol which attains the minimum. Further, let $W_{*}: \mathcal{X} \rightarrow \mathcal{Y}$ be a side-information channel such that $W_{*}\left(y_{*} \mid x\right)=1$ irrespective $x \in \mathcal{X}$. Then, let us consider a special case where $W_{1}=W_{*}$. This case is equivalent to the problem of "lossy coding when side-information may be absent". Heegard and Berger [12] (see also [25]) showed the following.

Proposition 4 ([[12]): We have

$$
R_{H B}\left(D_{1}, D_{2} \mid W_{*}, W_{2}\right)=\min \left[I\left(X ; \hat{X}_{1}\right)+I\left(X ; V \mid \hat{X}_{1}, Y\right)\right]
$$




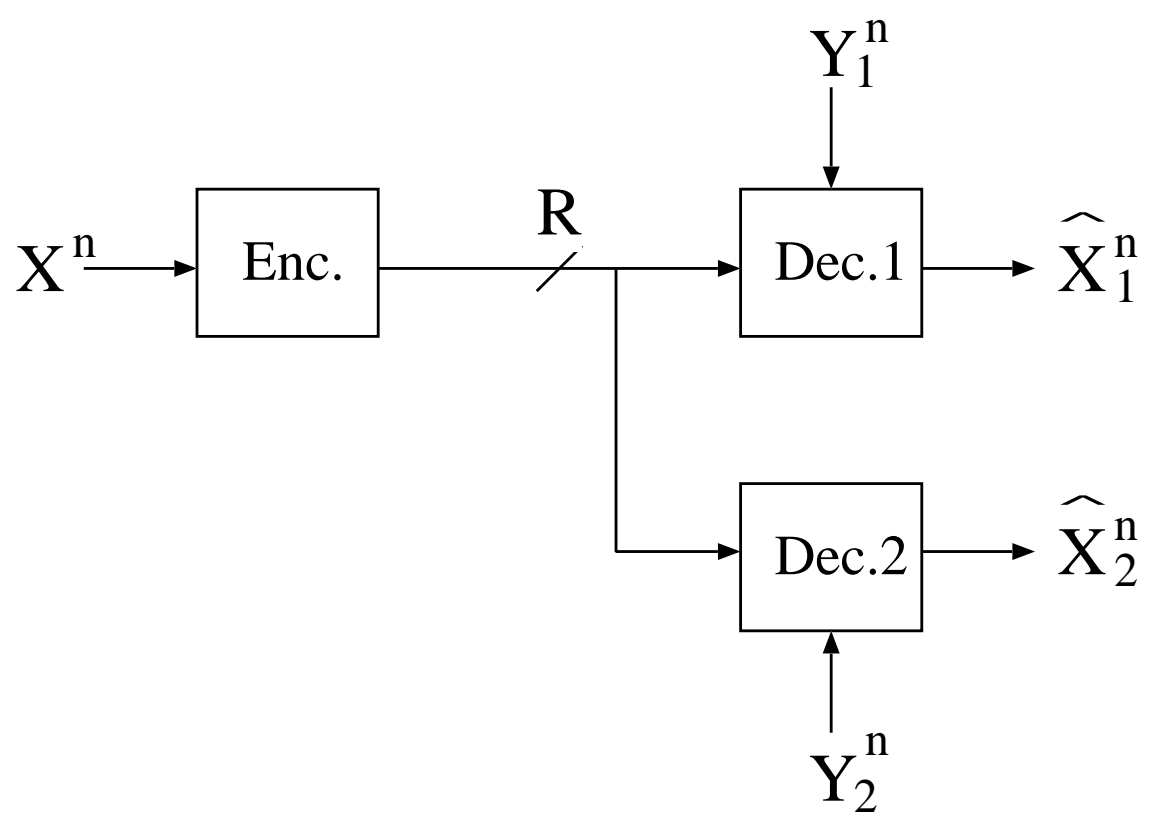

Fig. 2. The Heegard-Berger coding system.

where min is taken over all conditional distribution $P_{V \hat{X}_{1} \mid X}$ with $|\mathcal{V}| \leq|\mathcal{X} \times \hat{\mathcal{X}}|+2$ and functions $f: \mathcal{V} \times \hat{\mathcal{X}} \times \mathcal{Y} \rightarrow \hat{\mathcal{X}}$ such that

$$
\begin{aligned}
\mathbb{E}\left[d\left(X, X_{1}\right)\right] & \leq D_{1}, \\
\mathbb{E}\left[d\left(X, f\left(V, X_{1}, Y\right)\right)\right] & \leq D_{2} .
\end{aligned}
$$

\section{MAIN Result}

\section{A. Convex Form of WZ Rate-Distortion Function}

We need convex form of the Wyner-Ziv rate-distortion function introduced in [26]. Let $\mathcal{U}$ be the set of all functions from $\mathcal{Y}$ to $\hat{\mathcal{X}}$. The set $\mathcal{U}$ includes a constant function, i.e., $u(y)=\hat{x} \forall y \in \mathcal{Y}$ for each $\hat{x} \in \hat{\mathcal{X}}$. We denote the set of constant functions by $\overline{\mathcal{U}} \subset \mathcal{U}$. For fixed channel $W \in \mathcal{P}(\mathcal{Y} \mid \mathcal{X})$ and fixed test channel $V \in \mathcal{P}(\mathcal{U} \mid \mathcal{X})$, we denote

$$
d(V, W):=\sum_{u, x, y} P_{X}(x) V(u \mid x) W(y \mid x) d(x, u(y)) .
$$

For a fixed channel $W \in \mathcal{P}(\mathcal{Y} \mid \mathcal{X})$, let

$$
\mathcal{V}(W, D):=\{V \in \mathcal{P}(\mathcal{U} \mid \mathcal{X}): d(V, W) \leq D\} .
$$

Let

$$
\begin{aligned}
\mathcal{W}_{1}\left(P_{X}, E\right) & =\mathcal{W}_{1}(E) \\
& :=\left\{W \in \mathcal{P}(\mathcal{Y} \mid \mathcal{X}): e\left(P_{X}, W\right) \leq E\right\}
\end{aligned}
$$


and

$$
\mathcal{V}(E, D):=\left\{V \in \mathcal{P}(\mathcal{U} \mid \mathcal{X}): d(V, W) \leq D \forall W \in \mathcal{W}_{1}(E)\right\}
$$

For $(V, W) \in \mathcal{P}(\mathcal{U} \mid \mathcal{X}) \times \mathcal{P}(\mathcal{Y} \mid \mathcal{X})$, let

$$
\begin{aligned}
\phi(V, W) & :=I(U ; X)-I(U ; Y) \\
& =I(U ; X \mid Y) .
\end{aligned}
$$

Note that $\phi(\cdot, W)$ is a convex function for fixed $W$, which can be confirmed from (9), and $\phi(V, \cdot)$ is a concave function for fixed $V$, which can be confirmed from 8 ).

By the above notations, the Wyner-Ziv rate-distortion function is given by

$$
R_{W Z}(D \mid W)=\min _{V \in \mathcal{V}(W, D)} \phi(V, W)
$$

Let

$$
\tilde{R}_{W Z}(D \mid W, E)=\min _{V \in \mathcal{V}(E, D)} \phi(V, W)
$$

be the pseudo rate-distortion function.

Lemma 5: The pseudo rate-distortion function $\tilde{R}_{W Z}(D \mid W, E)$ is a concave function of the channel, i.e.,

$$
\begin{aligned}
& \tilde{R}_{W Z}\left(D \mid \lambda W_{1}+(1-\lambda) W_{2}, E\right) \\
& \quad \geq \lambda \tilde{R}_{W Z}\left(D \mid W_{1}, E\right)+(1-\lambda) \tilde{R}_{W Z}\left(D \mid W_{2}, E\right)
\end{aligned}
$$

holds for $W_{1}, W_{2} \in \mathcal{P}(\mathcal{Y} \mid \mathcal{X})$ and $0 \leq \lambda \leq 1$.

Proof: Let

$$
\hat{V}=\underset{V \in \mathcal{V}(E, D)}{\operatorname{argmin}} \phi\left(V, \lambda W_{1}+(1-\lambda) W_{2}\right) .
$$

Then, we have

$$
\begin{aligned}
R_{W Z}\left(D \mid \lambda W_{1}+(1-\lambda) W_{2}, E\right) \\
\quad=\phi\left(\hat{V}, \lambda W_{1}+(1-\lambda) W_{2}\right) \\
\geq \lambda \phi\left(\hat{V}, W_{1}\right)+(1-\lambda) \phi\left(\hat{V}, W_{2}\right) \\
\geq \lambda \min _{V \in \mathcal{V}(E, D)} \phi\left(V, W_{1}\right)+(1-\lambda) \min _{V \in \mathcal{V}(E, D)} \phi\left(V, W_{2}\right) \\
=\lambda R_{W Z}\left(D \mid W_{1}\right)+(1-\lambda) R_{W Z}\left(D \mid W_{2}\right),
\end{aligned}
$$

where we used concavity of $\phi(V, \cdot)$ for fixed $V$ in the first inequality. 


\section{B. Statements of General Results}

For the maximum distortion class, we have the following.

Theorem 6: We have

$$
\begin{aligned}
R_{m}(D \mid E) & \geq \max _{W \in \mathcal{W}_{1}(E)} R_{W Z}(D \mid W) \\
& =\max _{W \in \mathcal{W}_{1}(E)} \min _{V \in \mathcal{V}(W, D)} \phi(V, W)
\end{aligned}
$$

and

$$
\begin{aligned}
R_{m}(D \mid E) & \leq \min _{V \in \mathcal{V}(E, D)} \max _{W \in \mathcal{W}_{1}(E)} \phi(V, W) \\
& =\max _{W \in \mathcal{W}_{1}(E)} \min _{V \in \mathcal{V}(E, D)} \phi(V, W) \\
& =\max _{W \in \mathcal{W}_{1}(E)} \tilde{R}_{W Z}(D \mid W, E)
\end{aligned}
$$

Proof: See Section IV

Remark 7: Technically in the converse part, at $D=0$, we only have the inequality

$$
R_{m}(0 \mid E) \geq \lim _{\epsilon \downarrow 0} \max _{W \in \mathcal{W}_{1}(E)} R_{W Z}(\epsilon \mid W) .
$$

This is because we use the fact that $R_{W Z}(D \mid W)$ is a continuos function with respect to $W$ in the converse proof (see Section IV-A), and it is not clear whether $R_{W Z}(D \mid W)$ is a continuos function with respect to $W$ at $D=0$ in general.

The difference between (11) and (13) are $\mathcal{V}(W, D)$ and $\mathcal{V}(E, D)$. Thus, we have the following matching conditions.

Corollary 8: Let $\left(V^{*}, W^{*}\right)$ be a saddle point satisfying

$$
\phi\left(V^{*}, W^{*}\right)=\max _{W \in \mathcal{W}_{1}(E)} \min _{V \in \mathcal{V}(E, D)} \phi(V, W) .
$$

Suppose that

$$
\hat{V}:=\underset{V \in \mathcal{V}\left(W^{*}, D\right)}{\operatorname{argmin}} \phi\left(V, W^{*}\right) \in \mathcal{V}(E, D)
$$

Then, we have

$$
R_{m}(D \mid E)=\phi\left(V^{*}, W^{*}\right)=\max _{W \in \mathcal{W}_{1}(E)} \min _{V \in \mathcal{V}(E, D)} \phi(V, W)
$$

Proof: We have

$$
\begin{aligned}
\phi\left(\hat{V}, W^{*}\right) & =\min _{V \in \mathcal{V}\left(W^{*}, D\right)} \phi\left(V, W^{*}\right) \\
& \leq \max _{W \in \mathcal{W}_{1}(E)} \min _{V \in \mathcal{V}(W, D)} \phi(V, W) \\
& \leq R_{m}(D \mid E) \\
& \leq \phi\left(V^{*}, W^{*}\right) \\
& =\min _{V \in \mathcal{V}(E, D)} \phi\left(V, W^{*}\right) \\
& \leq \phi\left(\hat{V}, W^{*}\right) .
\end{aligned}
$$


Corollary 9: Under the same notations as Corollary 8, suppose that

$$
\operatorname{supp}(\hat{V}) \subset \overline{\mathcal{U}}
$$

Then, we have

$$
R_{m}(D \mid E)=\phi\left(V^{*}, W^{*}\right)=\max _{W \in \mathcal{W}_{1}(E)} \min _{V \in \mathcal{V}(E, D)} \phi(V, W)
$$

Proof: When (15) is satisfied, the distortion

$$
\sum_{u, x, y} P_{X}(x) \hat{V}(u \mid x) W(y \mid x) d(x, u(y))
$$

does not depend on the channel $W$. Thus, Corollary 8 implies the statement of the present corollary

For the average distortion class, we have the following.

Theorem 10: We have

$$
R_{a}(D \mid E) \geq \max _{\substack{\lambda, E_{1}, E_{2}, W_{1}, W_{2}: \\ \lambda \mathbb{1}_{1}+(1-\lambda) E_{2} \leq E \\ W_{1} \in \mathcal{W}_{1}\left(E_{1}\right), W_{2} \in \mathcal{W}_{1}\left(E_{2}\right)}} \min _{\substack{D_{1}, D_{2}: \\ \lambda D_{1}+(1-\lambda) D_{2} \leq D}} R_{H B}\left(D_{1}, D_{2} \mid W_{1}, W_{2}\right),
$$

where (i) max is taken over all $0 \leq \lambda \leq 1, E_{j} \geq 0$, and side information channels $W_{1}, W_{2}$ such that $\lambda E_{1}+$ $(1-\lambda) E_{2} \leq E$ and $W_{j} \in \mathcal{W}_{1}\left(E_{j}\right)(j=1,2)$ and (ii) min is taken over all $D_{1}, D_{2} \in\left[0, d_{\max }\right]$ such that $\lambda D_{1}+(1-\lambda) D_{2} \leq D$. Especially,

$$
R_{a}(D \mid E) \geq \max _{\substack{\lambda, E_{2}, W_{2} \in \mathcal{W}_{1}\left(E_{2}\right) \\ \lambda E_{*}+(1-\lambda) E_{2} \leq E}} \min _{\substack{D_{1}, D_{2}: \\ \lambda D_{1}+(1-\lambda) D_{2} \leq D}} R_{H B}\left(D_{1}, D_{2} \mid W_{*}, W_{2}\right)
$$

holds. We also have

$$
R_{a}(D \mid E) \leq \min _{V \in \mathcal{V}(E, D)} I\left(P_{X}, V\right)
$$

Proof: See Section $\mathrm{V}$

Remark 11: Note that (17) is obtained from (16) by letting $E_{1}=E_{*}$ and $W_{1}=W_{*}$. Thus, (16) is tighter than (17). However, we cannot give a single letter expression for the right hand side of (16), while we can for (17) by using Proposition 4

Remark 12: A close inspection of the proof reveals that we can generalize (16) by considering one-to- $m$ lossy source coding with side information at the decoders. That is, in the same manner as 16 , we can show that

$$
R_{a}(D \mid E) \geq \max _{\vec{\lambda}, \boldsymbol{E}, \boldsymbol{W}} \min _{\boldsymbol{D}} R_{H B}\left(D_{1}, D_{2}, \ldots, D_{m} \mid W_{1}, W_{2}, \ldots, W_{m}\right),
$$

where (i) max is taken over all $\vec{\lambda}=\left(\lambda_{1}, \ldots, \lambda_{m}\right), \boldsymbol{E}=\left(E_{1}, \ldots, E_{m}\right)$, and $\boldsymbol{W}=\left(W_{1}, \ldots, W_{m}\right)$ such that $\sum_{j} \lambda_{j}=1, \sum_{j} \lambda_{j} E_{j} \leq E$, and $W_{j} \in \mathcal{W}_{1}\left(E_{j}\right)(j=1, \ldots, m)$ and (ii) min is taken over all $\boldsymbol{D}=\left(D_{1}, \ldots, D_{m}\right)$ such that $\sum_{j} \lambda_{j} D_{j} \leq D$. The authors conjecture that the bound (19) is not tighter than (16), i.e., is equivalent to (16).

Remark 13: The upper bound in $(18)$ is derived by using the side-information only for the estimation at the decoder and not for the bin coding, which is the difference between (12) and (18). 
From Theorem 10, we have several corollaries. At first, let us set parameters in 17 as $\lambda=0$ and $E_{2}=E$. Then, we have

$$
\begin{aligned}
R_{a}(D \mid E) & \geq \max _{W_{2} \in \mathcal{W}_{1}(E)} \min _{D_{2} \leq D} R_{H B}\left(D_{1}, D_{2} \mid W_{*}, W_{2}\right) \\
& =\max _{W_{2} \in \mathcal{W}_{1}(E)} R_{H B}\left(d_{\max }, D \mid W_{*}, W_{2}\right) .
\end{aligned}
$$

Note that $R_{H B}\left(d_{\max }, D \mid W_{*}, W_{2}\right)$ equals to the Wyner-Ziv rate-distortion function $R_{W Z}\left(D \mid W_{2}\right)$. This fact gives the following corollary.

Corollary 14: We have

$$
R_{a}(D \mid E) \geq \max _{W \in \mathcal{W}_{1}(E)} R_{W Z}(D \mid W)
$$

Next, let us consider the lossless case, i.e., $d(\cdot, \cdot)$ is the Hamming distortion measure and $D=0$. Note that $R_{H B}\left(0,0 \mid W_{*}, W_{2}\right)$ equals to the minimum coding rate such that the decoder $\psi_{n}^{H B 1}$ without side information can reproduce $X^{n}$ in losslessly. Thus, for any side information channel $W_{2}$,

$$
R_{H B}\left(0,0 \mid W_{*}, W_{2}\right)=H(X) .
$$

Since $R_{a}(0 \mid E) \leq H(X)$, we have the following corollary.

Corollary 15: For $D=0$ and $E>0$, we have 1

$$
R_{a}(0 \mid E)=H(X)
$$

This corollary indicates that the side information is completely useless when $D=0$ and $E>0$. It should be emphasized that Corollary 14 does not give Corollary 15 in general. This means that our result (17) is tighter than Corollary 14

Lastly, we show that our bound (17) gives another trivial bound. Assume that $E \geq E_{*}$. Then, we can set $\lambda=1$ in (17) and have

$$
\begin{aligned}
R_{a}(D \mid E) & \geq \max _{W_{2} \in \mathcal{W}_{1}\left(E_{2}\right)} \min _{D_{1} \leq D} R_{H B}\left(D_{1}, D_{2} \mid W_{*}, W_{2}\right) \\
& =\max _{W_{2} \in \mathcal{W}_{1}\left(E_{2}\right)} R_{H B}\left(D, d_{\max } \mid W_{*}, W_{2}\right) .
\end{aligned}
$$

Furthermore, for any side information channel $W_{2}$, it is apparent that

$$
R_{H B}\left(D, d_{\max } \mid W_{*}, W_{2}\right) \geq R(D)
$$

where $R(D)$ is the rate-distortion function for one-to-one lossy coding without side information. Hence, if $E \geq E_{*}$, we have

$$
R_{a}(D \mid E) \geq R(D)
$$

\footnotetext{
${ }^{1}$ We need the condition $E>0$ because we need to take $\lambda>0$ in 17. When $e(\cdot, \cdot)$ is the Hamming distortion measure and $E=0$, then we have $R_{a}(0 \mid 0)=0$. However, $R_{a}(0 \mid 0)$ may be positive in general. For example, $R_{a}(0 \mid 0)$ can be positive for a distortion measure such that $e(x, y)=0$ for every $(x, y)$.
} 
Since $R_{a}(D \mid E) \leq R(D)$ always holds, we have the following corollary.

Corollary 16: If $E \geq E_{*}$, then we have

$$
R_{a}(D \mid E)=R(D)
$$

\section{Binary Hamming Example}

To provide some insight on our results, we consider the binary Hamming example, i.e., we assume that $\mathcal{X}=$ $\mathcal{Y}=\hat{\mathcal{X}}=\{0,1\}, P_{X}(0)=P_{X}(1)=\frac{1}{2}$, and

$$
\begin{aligned}
& e(x, y)= \begin{cases}0 & \text { if } x=y \\
1 & \text { else }\end{cases} \\
& d(x, \hat{x})= \begin{cases}0 & \text { if } x=\hat{x} \\
1 & \text { else }\end{cases}
\end{aligned}
$$

In this section, we assume that $E \leq \frac{1}{2}$.

We first consider the maximum distortion class. In this case, the set $\mathcal{W}_{1}(E)$ can be parametrized by two parameters $(\alpha, \beta)$ satisfying

$$
\frac{\alpha+\beta}{2} \leq E
$$

(see Fig. 3 and Fig. 4 ).

By the concavity of $\tilde{R}_{W Z}(D \mid W, E)$ with respect to $W$ (Lemma 5) and by the symmetry with respect to $\alpha$ and $\beta$, we have

$$
\underset{W \in \mathcal{W}_{1}(E)}{\operatorname{argmax}} \tilde{R}_{W Z}(D \mid W, E)=\mathrm{BSC}(E) .
$$

Let $0,1 \in \mathcal{U}$ be constant functions that output 0 or 1 irrespective of $y$ and let $y$ be the function that output $y$ itself. Similarly, let $\bar{y}$ be the function that outputs $y \oplus 1$. In the binary Hamming case, $\mathcal{U}=\{0,1, y, \bar{y}\}$. For $W^{*}=\operatorname{BSC}(E)$, it is known that

$$
R_{W Z}\left(D \mid W^{*}\right)=\min _{V \in \mathcal{V}\left(W^{*}, D\right)} \phi\left(V, W^{*}\right)
$$

is achieved by the test channel of the form

$$
\hat{V}(u \mid x)= \begin{cases}\lambda(1-q) & \text { if } u=x \\ \lambda q & \text { if } u=x \oplus 1 \\ (1-\lambda) & \text { if } u=y\end{cases}
$$

for some $0 \leq \lambda \leq 1$ and $0 \leq q \leq \frac{1}{2}$ ( $\lambda$ represents the time sharing). In this case, the distortion is given by

$$
\begin{aligned}
\lambda \sum_{\hat{x}, x} P_{X}(x) V_{q}(\hat{x} \mid x) d(x, \hat{x}) & \\
& +(1-\lambda) \sum_{x, y} P_{X}(x) W^{*}(y \mid x) d(x, y) \\
= & \lambda \sum_{\hat{x}, x} P_{X}(x) V_{q}(\hat{x} \mid x) d(x, \hat{x})+(1-\lambda) E \\
\quad \leq & D
\end{aligned}
$$




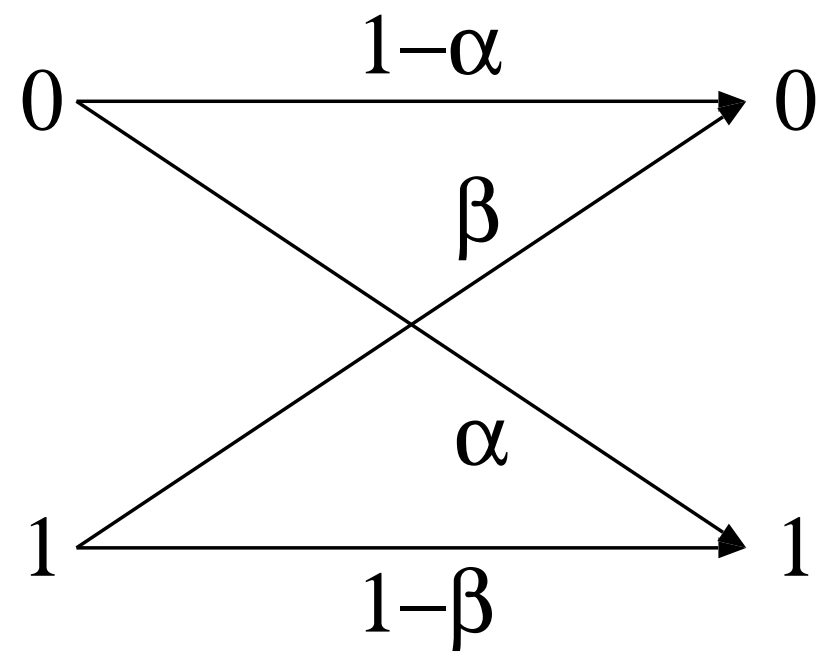

Fig. 3. Parametrization of binary channels.

where $V_{q}=\operatorname{BSC}(q)$. Since every channel $W \in \mathcal{W}_{1}(E)$ satisfies

$$
\begin{aligned}
& \sum_{x, y} P_{X}(x) W(y \mid x) d(x, y) \\
& \quad=\sum_{x, y} P_{X}(x) W(y \mid x) e(x, y) \\
& \leq E
\end{aligned}
$$

we find that $\hat{V} \in \mathcal{V}(E, D)$. Thus, the matching condition of Corollary 8 is satisfied for this binary Hamming example.

Next, we consider the average distortion class. We evaluate the upper bound (18). We first fix $W^{*}$ to be $\operatorname{BSC}(E)$. Note that

$$
\min _{V \in \mathcal{V}(E, D)} I\left(P_{X}, V\right) \geq \min _{V \in \mathcal{V}\left(W^{*}, D\right)} I\left(P_{X}, V\right) .
$$

For a test channel $V \in \mathcal{V}\left(W^{*}, D\right)$, let $\bar{V}$ be a test channel such that $\bar{V}(u \mid x)=V(u \oplus 1 \mid x \oplus 1)$ for $u \in \overline{\mathcal{U}}$ and $\bar{V}(u \mid x)=V(u \mid x \oplus 1)$ for $u \in\{y, \bar{y}\}$. Then, by the symmetry of the BSC and the source $P_{X}$, we have $\bar{V} \in \mathcal{V}\left(W^{*}, D\right)$ and $I\left(P_{X}, V\right)=I\left(P_{X}, \bar{V}\right)$. By the convexity of the mutual information for channel, we have

$$
I\left(P_{X}, \tilde{V}\right) \leq \frac{1}{2} I\left(P_{X}, V\right)+\frac{1}{2} I\left(P_{X}, \bar{V}\right),
$$

where $\tilde{V}=\frac{1}{2} V+\frac{1}{2} \bar{V}$. This means that the minimum in the right hand side of (21) is achieved by a symmetric test channel, i.e., $V(u \mid x)=V(u \oplus 1 \mid x \oplus 1)$ for $u \in \overline{\mathcal{U}}$ and $V(u \mid x)=V(u \mid x \oplus 1)$ for $u \in\{y, \bar{y}\}$. Furthermore, for $E \leq \frac{1}{2}$, we can assume that $V(\bar{y} \mid x)=0$ because using $\bar{y}$ only makes the distortion larger. We also note that such a symmetric test channel satisfies $V \in \mathcal{V}(E, D)$. Thus, the equality in 21) actually holds. Consequently, the upper bound on $R_{a}(D \mid E)$ in this example is the time sharing between the ordinary rate-distortion function and the distortion that can be achieved only by the estimation, i.e., the point $(E, 0)$. 


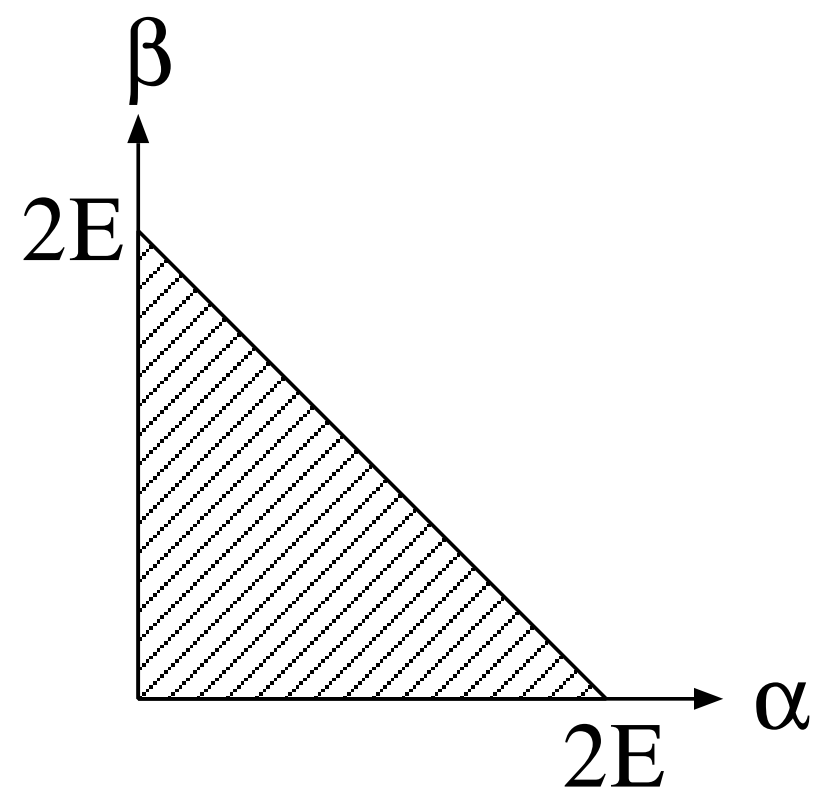

Fig. 4. The set of all channels in $\mathcal{W}_{1}(E)$.

\section{Discussion on Universality}

In this section, we discuss on the definitions of the universal Wyner-Ziv coding. We also discuss the relation between the universal Wyner-Ziv coding and the Heegard-Berger problem.

Let us consider the binary Hamming case as in the previous section. Let $X$ be the uniform random variable on $\{0,1\}$. Let $W_{1}$ be the binary channel in Fig. 3 with $\alpha=2 E$ and $\beta=0$, and let $W_{2}$ be the binary channel in Fig. 3 with $\alpha=0$ and $\beta=2 E$. Obviously, the Wyner-Ziv rate-distortion functions for $W_{1}$ and $W_{2}$ coincide, i.e.,

$$
R_{W Z}\left(D \mid W_{1}\right)=R_{W Z}\left(D \mid W_{2}\right)
$$

It should be also noted that $\mathcal{W}_{1}(E)$ is the convex hull of the set $\left\{W_{1}, W_{2}\right\}$.

As we have mentioned in Section $\amalg-\mathrm{B}$, in the ordinary definition of the universality, we require that there exists a universal code that works well for every $\mathcal{W}_{W Z}(R, D)$ instead of $\mathcal{W}_{1}(E)$. If we set $R=R_{W Z}\left(D \mid W_{1}\right)=$ $R_{W Z}\left(D \mid W_{2}\right)$, then we have

$$
W_{1}, W_{2} \in \mathcal{W}_{W Z}(R, D)
$$

Thus, at least, we have to construct a code that is universal for both $W_{1}$ and $W_{2}$, which can be regarded as a special case of the Heegard-Berger problem [12]. The rate-distortion function $R_{H B}\left(D, D \mid W_{1}, W_{2}\right)$ is not known, but we have a trivial lower bound

$$
\begin{aligned}
& R_{H B}\left(D, D \mid W_{1}, W_{2}\right) \\
& \quad \geq \quad R_{W Z}\left(D \mid W_{1}\right)=R_{W Z}\left(D \mid W_{2}\right) .
\end{aligned}
$$


The equality in (23) is a required condition such that the universal coding in the sense of $\mathcal{W}_{W Z}(R, D)$ to be possible. In other word, if the strict inequality holds in (23), this means that the universal coding in the sense of $\mathcal{W}_{W Z}(R, D)$ is impossible. Showing whether the equality holds or not is an important open problem.

A straightforward upper bound on $R_{H B}\left(D, D \mid W_{1}, W_{2}\right)$ can be derived as follows. Let $V_{s} \in \mathcal{P}(\mathcal{U} \mid \mathcal{X})$ be a symmetric test channel such that

$$
\begin{aligned}
V_{s}(0 \mid 0) & =V_{s}(1 \mid 1), \\
V_{s}(y \mid 0) & =V_{s}(y \mid 1), \\
V_{s}(\bar{y} \mid 0) & =V_{s}(\bar{y} \mid 1)=0 .
\end{aligned}
$$

Then, by taking $V_{s}(0 \mid 0)$ appropriately, we have

$$
V_{s} \in \mathcal{V}\left(W_{1}, D\right) \cap \mathcal{V}\left(W_{2}, D\right) .
$$

The achievability of

$$
\phi\left(V_{s}, W_{1}\right)=\phi\left(V_{s}, W_{2}\right)
$$

can be also derived from the known upper bound in [12]. Thus, we have

$$
\begin{aligned}
& R_{H B}\left(D, D \mid W_{1}, W_{2}\right) \\
& \quad \leq \quad \tilde{R}_{H B}\left(D, D \mid W_{1}, W_{2}\right) \\
& \quad:=\operatorname{lin}_{V_{s} \in \mathcal{V}\left(W_{1}, D\right) \cap \mathcal{V}\left(W_{2}, D\right)} \phi\left(V_{s}, W_{1}\right)
\end{aligned}
$$

Numerical calculations of $\tilde{R}_{H B}\left(D, D \mid W_{1}, W_{2}\right)$ and $R_{W Z}\left(D \mid W_{1}\right)$ are compared in Fig. 5. For comparison, we also plotted $R_{m}(D \mid E)$ in the figure. As we can find from the figure, $R_{m}(D \mid E)$ is much larger than $\tilde{R}_{H B}\left(D, D \mid W_{1}, W_{2}\right)$. This is because $\mathcal{W}_{1}(E)$ involves $\operatorname{BSC}(E)$.

\section{Proof of Theorem 6}

\section{A. Proof of Converse Part}

First we consider the case with $E=0$. Let $W \in \mathcal{W}_{1}(0)$. Then, from the definition of $\mathcal{W}_{m}(E)$, we have $\left\{W^{\times n}\right\}_{n=1}^{\infty} \in \mathcal{W}_{m}(0)$, which implies

$$
R_{m}(D \mid 0) \geq \max _{W \in \mathcal{W}_{1}(0)} R_{W Z}(D \mid W) .
$$

Next, we consider the case with $E>0$ and $D>0$. For any $0<\delta<E$, let $W \in \mathcal{W}_{1}(E-\delta)$. Then, from the definition of $\mathcal{W}_{m}(E)$, we have $\left\{W^{\times n}\right\}_{n=1}^{\infty} \in \mathcal{W}_{m}(E)$, which implies

$$
R_{m}(D \mid E) \geq \max _{W \in \mathcal{W}_{1}(E-\delta)} R_{W Z}(D \mid W) .
$$

Since this inequality holds for arbitrary $0<\delta<E$ and $R_{W Z}(D \mid W)$ is continuous with respect to $W$ for $D>0$, which will be proved in Appendix B, we have (10). 


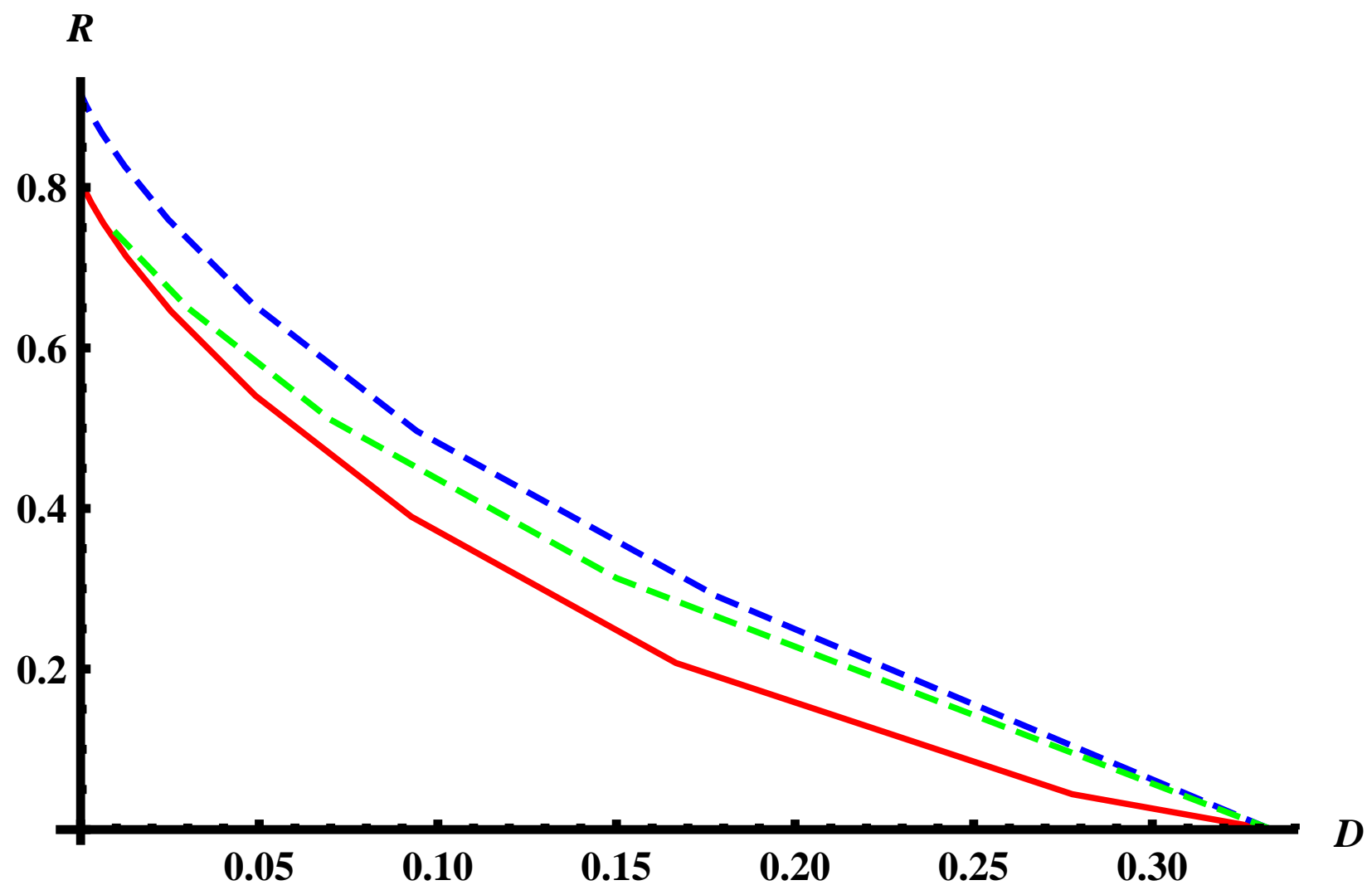

Fig. 5. Comparison among $\tilde{R}_{H B}\left(D, D \mid W_{1}, W_{2}\right), R_{W Z}\left(D \mid W_{1}\right)$, and $R_{m}(D \mid E)$. The red solid line is $R_{W Z}\left(D \mid W_{1}\right)$. The green dashed line is $\tilde{R}_{H B}\left(D, D \mid W_{1}, W_{2}\right)$. The blue dashed line is $R_{m}(D \mid E)$.

When $E>0$ and $D=0$, for $\epsilon>0$, we first prove

$$
\begin{aligned}
R_{m}(0 \mid E) & \geq \max _{W \in \mathcal{W}_{1}(E-\delta)} R_{W Z}(0 \mid W) \\
& \geq \max _{W \in \mathcal{W}_{1}(E-\delta)} R_{W Z}(\epsilon \mid W)
\end{aligned}
$$

Then, by the continuity argument, we have

$$
R_{m}(0 \mid E) \geq \lim _{\epsilon \downarrow 0} \max _{W \in \mathcal{W}_{1}(E)} R_{W Z}(\epsilon \mid W)
$$

\section{B. Proof of Direct Part}

Note that the function $\phi(\cdot, W)$ is a convex function for fixed $W, \phi(V, \cdot)$ is a concave function for fixed $V$, and $\mathcal{W}_{1}(E)$ and $\mathcal{V}(E, D)$ are convex sets. Thus, (13) is derived from (12) by applying the saddle point theorem [27]. We prove (12) by three steps. First, we prove that there exists a universal code for i.i.d. channels. Then, we show that there exists a randomized universal code for permutation invariant channels. Finally, we de-randomize the randomized universal code by using the technique of [28], [29]. 
1) Code for i.i.d. Channel: In this section, we construct a universal Wyner-Ziv code for a fixed test channel such that it works well for every $W \in \mathcal{W}_{1}(E) \cap \mathcal{P}_{n}(\mathcal{Y} \mid \mathcal{X})$. We construct a universal Wyner-Ziv code by using the output statistics of random binning argument recently introduced by [30]. We note that a universal Wyner-Ziv code can be also constructed from the coding method in [31].

Let us fix $V \in \mathcal{V}(D, E)$. We use two kinds of bin codings $f_{n}: \mathcal{U}^{n} \rightarrow \mathcal{S}_{n}$ and $g_{n}: \mathcal{U}^{n} \rightarrow \mathcal{L}_{n}$. Let $F_{n}$ and $G_{n}$ be random bin codings. For arbitrary small $\delta>0$, let $R_{f}, R_{g} \geq 0$ be the real numbers such that

$$
\begin{aligned}
R_{f} & =H(U \mid X)-\delta, \\
R_{g} & =\max _{W \in \mathcal{W}_{1}(E)} \phi(V, W)+2 \delta \\
& =\max _{W \in \mathcal{W}_{1}(E)} I(U ; X \mid Y)+2 \delta .
\end{aligned}
$$

Since

$$
\begin{aligned}
I(U ; X \mid Y) & =H(U \mid Y)-H(U \mid X, Y) \\
& =H(U \mid Y)-H(U \mid X)
\end{aligned}
$$

we have

$$
R_{f}+R_{g}=\max _{W \in \mathcal{W}_{1}(E)} H(U \mid Y)+\delta .
$$

Let $\left|\mathcal{S}_{n}\right|=\left\lfloor 2^{n R_{f}}\right\rfloor$ and $\left|\mathcal{L}_{n}\right|=\left\lceil 2^{n R_{g}}\right\rceil$.

From (27), we find that the sum rate $R_{f}+R_{g}$ is sufficiently large for the Slepian-Wolf coding. We use the following lemma on universal Slepian-Wolf coding.

Lemma 17: For sufficiently large $n$, there exists $\mu_{1}>0$ and a universal decoder $\kappa_{n}: \mathcal{Y}^{n} \times \mathcal{S}_{n} \times \mathcal{L}_{n} \rightarrow \mathcal{U}^{n}$ such that

$$
\mathbb{E}_{F_{n} G_{n}}\left[P_{\text {err }}\left(F_{n}, G_{n}, W\right)\right] \leq 2^{-\mu_{1} n}
$$

for every $W \in \mathcal{W}_{1}(E) \cap \mathcal{P}_{n}(\mathcal{Y} \mid \mathcal{X})$, where $P_{\text {err }}\left(F_{n}, G_{n}, W\right)$ is the error probability of the Slepian-Wolf coding for channel $W$ when the bin codings $\left(F_{n}, G_{n}\right)$ are used.

Proof: The lemma is proved exactly in the same manner as [4]. A few modifications are that we use the random bin coding instead of the random linear coding 2 , and that we evaluate the ensemble average of the error probability.

From (24), we find that the rate $R_{f}$ is sufficiently small to generate the uniform random variable that is independent of $X^{n}$. We use the privacy amplification lemma (Lemma 30) described in Appendix D

We construct a code as follows. Let

$$
P_{\hat{U}^{n} \mid Y^{n} S_{n} L_{n}}\left(u^{n} \mid y^{n}, s_{n}, \ell_{n}\right)=\mathbf{1}\left[u^{n}=\kappa_{n}\left(y^{n}, s_{n}, \ell_{n}\right)\right]
$$

\footnotetext{
${ }^{2}$ we can also use the random linear coding instead of the random bin coding because Lemma 30 holds under the condition that $f_{n}$ is chosen from a universal hash family and the random linear coding ensemble is a universal hash family.
} 
be the distribution describing the Slepian-Wolf decoder. Let

$$
\begin{aligned}
& P_{S_{n} L_{n} U^{n} X^{n} Y^{n} \hat{U}^{n}}\left(s_{n}, \ell_{n}, u^{n}, x^{n}, y^{n}, \hat{u}^{n}\right) \\
& =P_{S_{n} X^{n}}\left(s_{n}, x^{n}\right) P_{U^{n} \mid S_{n} X^{n}}\left(u^{n} \mid s_{n}, x^{n}\right) P_{L_{n} \mid U^{n}}\left(\ell_{n} \mid u^{n}\right) \\
& \quad P_{Y^{n} \mid X^{n}}\left(y^{n} \mid x^{n}\right) P_{\hat{U}^{n} \mid Y^{n} S_{n} L_{n}}\left(\hat{u}^{n} \mid y^{n}, s_{n}, \ell_{n}\right)
\end{aligned}
$$

and

$$
\begin{aligned}
\hat{P}_{\bar{S}_{n} L_{n} U^{n} X^{n} Y^{n} \hat{U}^{n}}\left(s_{n}, \ell_{n}, u^{n}, x^{n}, y^{n}, \hat{u}^{n}\right) & \\
= & P_{\bar{S}_{n}}\left(s_{n}\right) P_{X^{n}}\left(x^{n}\right) P_{U^{n} \mid S_{n} X^{n}}\left(u^{n} \mid s_{n}, x^{n}\right) P_{L_{n} \mid U^{n}}\left(\ell_{n} \mid u^{n}\right) \\
& P_{Y^{n} \mid X^{n}}\left(y^{n} \mid x^{n}\right) P_{\hat{U}^{n} \mid Y^{n} S_{n} L_{n}}\left(\hat{u}^{n} \mid y^{n}, s_{n}, \ell_{n}\right) .
\end{aligned}
$$

The distribution $P_{S_{n} L_{n} U^{n} X^{n} Y^{n} \hat{U}^{n}}$ describes a virtual coding scheme in which the encoder sends both $F_{n}\left(U^{n}\right)$ and $G_{n}\left(U^{n}\right)$. The distribution $\hat{P}_{\bar{S}_{n} L_{n} U^{n} X^{n} Y^{n} \hat{U}^{n}}$ describes a real coding scheme in which the encoder sends only $G_{n}\left(U^{n}\right)$ and uses the common randomness $\bar{S}_{n}$ that is shared with the decoder. Note that $P_{U^{n} \mid S_{n} X^{n}}\left(u^{n} \mid s_{n}, x^{n}\right)$ is a randomized quantizer, which is derived from the bin coding $f_{n}$ and the test channel $P_{U \mid X}$ via $P_{U^{n} X^{n} S_{n}}$. From Lemma 30 and the fact that the variational distance does not increase by data processing or marginalization, we have

$$
\mathbb{E}_{F_{n} G_{n}}\left[\left\|\hat{P}_{\bar{S}_{n} U^{n} X^{n} Y^{n} \hat{U}^{n}}-P_{S_{n} U^{n} X^{n} Y^{n} \hat{U}^{n}}\right\|\right] \leq 2^{-\mu_{2} n}
$$

for some $\mu_{2}>0$. By the large deviation bound such as the Bernstein inequality, there exists $\mu_{3}>0$ such that

$$
P_{U^{n} X^{n} Y^{n}}\left(\left\{d_{n}\left(x^{n}, u^{n}\left(y^{n}\right)\right)>D+\delta\right\}\right) \leq 2^{-\mu_{3} n} .
$$

It should be noted that the bound 28 is uniform with respect to the channel $W$. By Lemma 17 we have

$$
\begin{aligned}
& \mathbb{E}_{F_{n} G_{n}}\left[P_{S_{n} U^{n} X^{n} Y^{n} \hat{U}^{n}}\left(\left\{d_{n}\left(x^{n}, \hat{u}^{n}\left(y^{n}\right)\right)>D+\delta\right\}\right)\right] \\
& \leq \mathbb{E}_{F_{n} G_{n}}\left[P _ { S _ { n } U ^ { n } X ^ { n } Y ^ { n } \hat { U } ^ { n } } \left(\left\{d_{n}\left(x^{n}, u^{n}\left(y^{n}\right)\right)>D+\delta\right.\right.\right. \\
& \left.\left.\left.\quad \text { or } u^{n} \neq \hat{u}^{n}\right\}\right)\right] \\
& \leq 2^{-\mu_{1} n}+2^{-\mu_{3} n} .
\end{aligned}
$$

Since

$$
\begin{aligned}
& \hat{P}_{\bar{S}_{n} U^{n} X^{n} Y^{n} \hat{U}^{n}}(\mathcal{A})-P_{S_{n} U^{n} X^{n} Y^{n} \hat{U}^{n}}(\mathcal{A}) \\
& \quad \leq\left\|\hat{P}_{\bar{S}_{n} U^{n} X^{n} Y^{n} \hat{U}^{n}}-P_{S_{n} U^{n} X^{n} Y^{n} \hat{U}^{n}}\right\|
\end{aligned}
$$

for any set $\mathcal{A}$, we have

$$
\begin{aligned}
& \mathbb{E}_{F_{n} G_{n}}\left[\hat{P}_{\bar{S}_{n} U^{n} X^{n} Y^{n} \hat{U}^{n}}\left(\left\{d_{n}\left(x^{n}, \hat{u}^{n}\left(y^{n}\right)\right)>D+\delta\right\}\right)\right] \\
& \quad \leq 3 \cdot 2^{-n \min \mu_{i}} .
\end{aligned}
$$


Since

$$
\left|\mathcal{W}_{1}(E) \cap \mathcal{P}_{n}(\mathcal{Y} \mid \mathcal{X})\right| \leq(n+1)^{|\mathcal{X}||\mathcal{Y}|},
$$

there exists at least one realization $\left(f_{n}, g_{n}, s_{n}\right)$ of $\left(F_{n}, G_{n}, S_{n}\right)$ such that

$$
\begin{aligned}
& \hat{P}_{U^{n} X^{n} Y^{n} \hat{U}^{n} \mid \bar{S}_{n}}\left(\left\{d_{n}\left(x^{n}, \hat{u}^{n}\left(y^{n}\right)\right)>D+\delta\right\} \mid s_{n}\right) \\
& \quad \leq 3(n+1)^{|\mathcal{X}||\mathcal{Y}|} 2^{-n \min \mu_{i}} .
\end{aligned}
$$

for every $W \in \mathcal{W}_{1}(E) \cap \mathcal{P}_{n}(\mathcal{Y} \mid \mathcal{X})$. Furthermore, let $K_{n}$ be a random variable that simulate the randomized quantizer $P_{U^{n} \mid S_{n} X^{n}}$. Then, we can also eliminate this randomness in a similar manner as above.

In summary, we have shown the following.

Lemma 18: For any $V \in \mathcal{V}(E, D)$ and any $\delta>0$, there exists a universal code $\left(\varphi_{n}, \psi_{n}\right)$ and a constant $\mu>0$ such that

$$
\frac{1}{n} \log \left|\mathcal{M}_{n}\right| \leq \max _{W \in \mathcal{W}_{1}(E)} \phi(V, W)+2 \delta
$$

and

$$
\operatorname{Pr}\left\{d_{n}\left(X^{n}, \psi_{n}\left(\varphi_{n}\left(X^{n}\right), Y^{n}\right)\right)>D+\delta\right\} \leq 2^{-\mu n}
$$

for every $W \in \mathcal{W}_{1}(E) \cap \mathcal{P}_{n}(\mathcal{Y} \mid \mathcal{X})$ provided that $n$ is sufficiently large.

2) Code for Permutation Invariant Channel: In Section IV-B1 we constructed a universal Wyner-Ziv code $\left(\varphi_{n}, \psi_{n}\right)$ for a fixed test channel such that it works well for every $W \in \mathcal{W}_{1}(E) \cap \mathcal{P}_{n}(\mathcal{Y} \mid \mathcal{X})$. In this section, we use this code to the channel in $\mathcal{W}_{m}(E)$. Let $\pi_{n}$ be random permutation on $\{1, \ldots, n\}$. We first apply the random permutation to the sequence $\left(X^{n}, Y^{n}\right)$ and then use $\left(\varphi_{n}, \psi_{n}\right)$. It should be noted that the encoder and the decoder agree with a realization of the random permutation in this section. We denote

$$
P_{X}^{n} \cdot W^{n}\left(x^{n}, y^{n}\right)=P_{X}^{n}\left(x^{n}\right) W^{n}\left(y^{n} \mid x^{n}\right) .
$$

Note that

$$
\begin{aligned}
\mathbb{E}_{\pi_{n}} & {\left[P_{X}^{n} \cdot W^{n}\left(\pi_{n}\left(x^{n}\right), \pi_{n}\left(y^{n}\right)\right)\right] } \\
& =\mathbb{E}_{\pi_{n}}\left[P_{X}^{n}\left(\pi_{n}\left(x^{n}\right) W^{n}\left(\pi_{n}\left(y^{n}\right) \mid \pi_{n}\left(x^{n}\right)\right)\right]\right. \\
& =P_{X}^{n}\left(x^{n}\right) \mathbb{E}_{\pi_{n}}\left[W^{n}\left(\pi_{n}\left(y^{n}\right) \mid \pi_{n}\left(x^{n}\right)\right)\right],
\end{aligned}
$$

and we consider the average performance with respect to the permutation. Thus, without loss of generality, we can assume that $W^{n}$ is permutation invariant, i.e., $W^{n}\left(y^{n} \mid x^{n}\right)=W^{n}\left(\tilde{y}^{n} \mid \tilde{x}^{n}\right)$ if $P_{x^{n}} y^{n}=P_{\tilde{x}^{n}} \tilde{y}^{n}$.

Lemma 19: Let $\mathcal{A}_{n} \subset \mathcal{X}^{n} \times \mathcal{Y}^{n}$. Suppose that

$$
P_{X}^{n} \cdot \bar{W}^{\times n}\left(\mathcal{A}_{n}^{c}\right) \leq \bar{\varepsilon}
$$


for every $\bar{W} \in \mathcal{W}_{1}\left(E+\delta e_{\max }\right) \cap \mathcal{P}_{n}(\mathcal{Y} \mid \mathcal{X})$. Then, for any conditional type $\bar{W} \in \overline{\mathcal{W}}_{n}\left(T_{X, \delta}, E\right)$, we have

$$
\begin{aligned}
& \sum_{x^{n} \in T_{X, \delta}} P_{X}^{n}\left(x^{n}\right) \mathbf{1}\left[\bar{W} \in \mathcal{P}_{n}\left(\mathcal{Y} \mid P_{x^{n}}\right)\right] \\
& \quad \times \sum_{y^{n} \in T_{\bar{W}}\left(x^{n}\right)} \frac{1}{\left|T_{\bar{W}}\left(x^{n}\right)\right|} \mathbf{1}\left[\left(x^{n}, y^{n}\right) \in \mathcal{A}_{n}^{c}\right] \\
& \leq(n+1)^{|\mathcal{X}||\mathcal{Y}|} \bar{\varepsilon} .
\end{aligned}
$$

Proof: For any $\bar{W} \in \mathcal{P}_{n}\left(\mathcal{Y} \mid P_{x^{n}}\right)$, note that

$$
\begin{aligned}
\bar{W}^{\times n}\left(T_{\bar{W}}\left(x^{n}\right) \mid x^{n}\right) & \geq \frac{1}{(n+1)^{|\mathcal{X}||\mathcal{Y}|}} 2^{-n D\left(\bar{W} \| \bar{W} \mid P_{x} n\right)} \\
& =\frac{1}{(n+1)^{|\mathcal{X}||\mathcal{Y}|}} .
\end{aligned}
$$

From [51, we have

$$
\bar{W} \in \overline{\mathcal{W}}_{n}\left(T_{X, \delta}, E\right) \Longrightarrow \bar{W} \in \mathcal{W}_{1}\left(E+\delta e_{\max }\right)
$$

Thus, for every $\bar{W} \in \overline{\mathcal{W}}_{n}\left(T_{X, \delta}, E\right)$ we have

$$
\begin{aligned}
\bar{\varepsilon} \geq & P_{X}^{n} \cdot \bar{W}^{\times n}\left(\mathcal{A}_{n}^{c}\right) \\
\geq & \sum_{x^{n} \in T_{X, \delta}} P_{X}^{n}\left(x^{n}\right) \mathbf{1}\left[\bar{W} \in \mathcal{P}_{n}\left(\mathcal{Y} \mid P_{x^{n}}\right)\right] \sum_{y^{n} \in T_{\bar{W}}\left(x^{n}\right)} \\
& \times \bar{W}^{\times n}\left(T_{\bar{W}}\left(x^{n}\right) \mid x^{n}\right) \frac{1}{\left|T_{\bar{W}}\left(x^{n}\right)\right|} \mathbf{1}\left[\left(x^{n}, y^{n}\right) \in \mathcal{A}_{n}^{c}\right] \\
\geq & \frac{1}{(n+1)^{|\mathcal{X}||\mathcal{Y}|}} \sum_{x^{n} \in T_{X, \delta}} P_{X}^{n}\left(x^{n}\right) \mathbf{1}\left[\bar{W} \in \mathcal{P}_{n}\left(\mathcal{Y} \mid P_{x^{n}}\right)\right] \\
& \times \sum_{y^{n} \in T_{\bar{W}}\left(x^{n}\right)} \frac{1}{\left|T_{\bar{W}}\left(x^{n}\right)\right|} \mathbf{1}\left[\left(x^{n}, y^{n}\right) \in \mathcal{A}_{n}^{c}\right],
\end{aligned}
$$

which implies the statement of the lemma.

Lemma 20: Suppose that the code $\left(\varphi_{n}, \psi_{n}\right)$ satisfies

$$
\operatorname{Pr}\left\{d_{n}\left(X^{n}, \psi_{n}\left(\varphi_{n}\left(X^{n}\right), Y^{n}\right)\right)>D+\delta\right\} \leq \bar{\varepsilon}
$$

for every i.i.d. channel $\bar{W}^{\times n}$ such that $\bar{W} \in \mathcal{W}_{1}\left(E+\delta e_{\max }\right) \cap \mathcal{P}_{n}(\mathcal{Y} \mid \mathcal{X})$, where $\left(X^{n}, Y^{n}\right) \sim P_{X}^{n} \cdot \bar{W}^{\times n}$. Then, we have

$$
\begin{aligned}
& \operatorname{Pr}\left\{d_{n}\left(X^{n}, \psi_{n}\left(\varphi_{n}\left(X^{n}\right), Y^{n}\right)\right)>D+\delta\right\} \\
& \leq(n+1)^{2|\mathcal{X}||\mathcal{Y}|} \bar{\varepsilon}+P_{X}^{n}\left(\left(T_{X, \delta}^{n}\right)^{c}\right)+\delta_{1}
\end{aligned}
$$

for every permutation invariant (not necessarily i.i.d.) $W^{n}$ satisfying

$$
\operatorname{Pr}\left\{e_{n}\left(X^{n}, Y^{n}\right)>E\right\} \leq \delta_{1},
$$

where $\left(X^{n}, Y^{n}\right) \sim P_{X}^{n} \cdot W^{n}$. 
Proof: Suppose that $\left(X^{n}, Y^{n}\right) \sim P_{X}^{n} \cdot W^{n}$. By using Lemma 19 for

$$
\mathcal{A}_{n}:=\left\{\left(x^{n}, y^{n}\right): d_{n}\left(x^{n}, \psi_{n}\left(\varphi_{n}\left(x^{n}\right), y^{n}\right)\right) \leq D+\delta\right\},
$$

we have

$$
\begin{aligned}
& \operatorname{Pr}\left\{d_{n}\left(X^{n}, \psi_{n}\left(\varphi_{n}\left(X^{n}\right), Y^{n}\right)\right)>D+\delta\right\} \\
& \leq \sum_{x^{n} \notin T_{X, \delta}} P_{X}^{n}\left(x^{n}\right) \\
& +\sum_{\bar{W} \in \overline{\mathcal{W}}_{n}\left(T_{X, \delta}, E\right)} \sum_{x^{n} \in T_{X, \delta}} P_{X}^{n}\left(x^{n}\right) \mathbf{1}\left[\bar{W} \in \mathcal{P}_{n}\left(\mathcal{Y} \mid P_{x^{n}}\right)\right] \\
& \times \sum_{y^{n} \in T_{\bar{W}}\left(x^{n}\right)} W^{n}\left(T_{\bar{W}}\left(x^{n}\right) \mid x^{n}\right) \frac{1}{\left|T_{\bar{W}}\left(x^{n}\right)\right|} \mathbf{1}\left[\left(x^{n}, y^{n}\right) \in \mathcal{A}_{n}^{c}\right] \\
& +\sum_{\bar{W} \notin \overline{\mathcal{W}}_{n}\left(T_{X, \delta}, E\right)} \sum_{x^{n} \in T_{X, \delta}} P_{X}^{n}\left(x^{n}\right) \mathbf{1}\left[\bar{W} \in \mathcal{P}_{n}\left(\mathcal{Y} \mid P_{x^{n}}\right)\right] W^{n}\left(T_{\bar{W}}\left(x^{n}\right) \mid x^{n}\right) \\
& \leq P_{X}^{n}\left(T_{X, \delta}^{c}\right) \\
& +\sum_{\bar{W} \in \overline{\mathcal{W}}_{n}\left(T_{X, \delta}, E\right)} \sum_{x^{n} \in T_{X, \delta}} P_{X}^{n}\left(x^{n}\right) \mathbf{1}\left[\bar{W} \in \mathcal{P}_{n}\left(\mathcal{Y} \mid P_{x^{n}}\right)\right] \\
& \times \sum_{y^{n} \in T_{\bar{W}}\left(x^{n}\right)} \frac{1}{\left|T_{\bar{W}}\left(x^{n}\right)\right|} \mathbf{1}\left[\left(x^{n}, y^{n}\right) \in \mathcal{A}_{n}^{c}\right] \\
& +\operatorname{Pr}\left\{e_{n}\left(X^{n}, Y^{n}\right)>E\right\} \\
& \leq(n+1)^{2|\mathcal{X}||\mathcal{Y}|} \bar{\varepsilon}+P_{X}^{n}\left(T_{X, \delta}^{c}\right)+\delta_{1},
\end{aligned}
$$

where we used $W^{n}\left(T_{\bar{W}}\left(x^{n}\right) \mid x^{n}\right) \leq 1$ to bound the second term, we used the fact

$$
\bar{W} \notin \overline{\mathcal{W}}_{n}\left(P_{x^{n}}, E\right) \Longleftrightarrow e\left(P_{x^{n}}, \bar{W}\right)>E
$$

and (49) to bound the third term in the second inequality, and we used Lemma 19 to bound the second term in the third inequality.

By combining Lemma 18 and Lemma 20 and by noting the definition of $\mathcal{W}_{m}(E)$, we have the following.

Lemma 21: For any $V \in \mathcal{V}\left(E+\delta e_{\max }, D\right)$, any $\delta>0$, and any $\varepsilon>0$, there exists a universal code $\left(\varphi_{n}, \psi_{n}\right)$ such that

$$
\frac{1}{n} \log \left|\mathcal{M}_{n}\right| \leq \max _{W \in \mathcal{W}_{1}\left(E+\delta e_{\max }\right)} \phi(V, W)+2 \delta
$$

and

$$
\begin{aligned}
& \mathbb{E}_{\pi_{n}}\left[\operatorname{Pr}\left\{d_{n}\left(\pi_{n}\left(X^{n}\right), \psi_{n}\left(\varphi_{n}\left(\pi_{n}\left(X^{n}\right)\right), \pi_{n}\left(Y^{n}\right)\right)\right)>D+\delta\right\}\right] \\
& \quad \leq \varepsilon
\end{aligned}
$$

for every $\mathbf{W} \in \mathcal{W}_{m}(E)$ provided that $n$ is sufficiently large. 
3) De-Randomization: Now we reduce the size of random permutation by using the de-randomization technique.

Lemma 22: Suppose that $\left(\varphi_{n}, \psi_{n}\right)$ satisfies (31). Then, for arbitrary $\delta_{2}, \gamma>0$, there exists $m_{n}=2^{\delta_{2} n}$ permutations $\left\{\pi_{n}^{(1)}, \ldots, \pi_{n}^{\left(m_{n}\right)}\right\}$ such that

$$
\begin{gathered}
\frac{1}{m_{n}} \sum_{i=1}^{m_{n}} \operatorname{Pr}\left\{d_{n}\left(\pi_{n}^{(i)}\left(X^{n}\right), \psi_{n}\left(\varphi_{n}\left(\pi_{n}^{(i)}\left(X^{n}\right)\right), \pi_{n}^{(i)}\left(Y^{n}\right)\right)\right)\right. \\
>D+\delta\} \leq \varepsilon+\gamma
\end{gathered}
$$

provided that $n$ is sufficiently large.

Proof: For a permutation $\pi_{n}$ and $\left(x^{n}, y^{n}\right) \in \mathcal{X}^{n} \times \mathcal{Y}^{n}$, we denote

$$
\begin{aligned}
& I\left(\pi_{n}, x^{n}, y^{n}\right) \\
& \quad=\mathbf{1}\left[d_{n}\left(\pi_{n}\left(x^{n}\right), \psi_{n}\left(\varphi_{n}\left(\pi_{n}\left(x^{n}\right)\right), \pi_{n}\left(y^{n}\right)\right)\right)>D+\delta\right] .
\end{aligned}
$$

Let $\pi_{n}^{(1)}, \ldots, \pi_{n}^{\left(m_{n}\right)}$ be randomly generated permutations, and let $\bar{I}\left(x^{n}, y^{n}\right)=\mathbb{E}_{\pi_{n}}\left[I\left(\pi_{n}, x^{n}, y^{n}\right)\right]$. Then, by using Lemma 31 for $A_{i}=I\left(\pi_{n}^{(i)}, x^{n}, y^{n}\right), b=1$, and $\alpha=\frac{\gamma}{2}$, we have

$$
\begin{aligned}
& \operatorname{Pr}\left\{\frac{1}{m_{n}} \sum_{i=1}^{m_{n}} I\left(\pi_{n}^{(i)}, x^{n}, y^{n}\right) \geq \bar{I}\left(x^{n}, y^{n}\right)+\gamma\right\} \\
& \leq \exp \left\{-\left(\gamma^{2} / 4\right) m_{n}\right\} .
\end{aligned}
$$

Furthermore, by using the union bound, we have

$$
\begin{aligned}
& \operatorname{Pr}\left\{\exists\left(x^{n}, y^{n}\right) \frac{1}{m_{n}} \sum_{i=1}^{m_{n}} I\left(\pi_{n}^{(i)}, x^{n}, y^{n}\right) \geq \bar{I}\left(x^{n}, y^{n}\right)+\gamma\right\} \\
& \leq\left|\mathcal{X}^{n}\right|\left|\mathcal{Y}^{n}\right| \exp \left\{-\left(\gamma^{2} / 4\right) m_{n}\right\} .
\end{aligned}
$$

Since $\exp \left\{-\left(\gamma^{2} / 4\right) m_{n}\right\}$ converges to 0 doubly exponentially, the right hand side of 32 is strictly smaller than 1 if $n$ is sufficiently large, which implies that there exists one realization of $\pi_{1}^{(1)}, \ldots, \pi_{n}^{\left(m_{n}\right)}$ such that

$$
\frac{1}{m_{n}} \sum_{i=1}^{m_{n}} I\left(\pi_{n}^{(i)}, x^{n}, y^{n}\right) \leq \bar{I}\left(x^{n}, y^{n}\right)+\gamma
$$

for every $\left(x^{n}, y^{n}\right)$. Finally, by taking the average of both sides of 33 with respect to $\left(X^{n}, Y^{n}\right)$, we have the assertion of the lemma.

Finally, by combining Lemma 21 and Lemma 22 , by taking the constants to be sufficiently small and $n$ to be sufficiently large, we can show [12).

\section{Proof of Theorem 10}

\section{A. Proof of Converse Part}

We only prove (16) because (17) is obtained from (16) by letting $E_{1}=E_{*}$ and $W_{1}=W_{*}$.

Assume that $R$ is achievable and fix $\lambda, E_{1}, E_{2}, W_{1}$, and $W_{2}$ such that $\lambda E_{1}+(1-\lambda) E_{2} \leq E$ and $W_{j} \in \mathcal{W}_{j}\left(E_{j}\right)$ for $j=1,2$. To prove (16), it is sufficient to show that there exists a pair $\left(D_{1}, D_{2}\right)$ such that $\lambda D_{1}+(1-\lambda) D_{2} \leq D$ and $R \geq R_{H B}\left(D_{1}, D_{2} \mid W_{1}, W_{2}\right)$. 
To do this, we consider the compound channel

$$
W^{n}=\lambda W_{1}^{\times n}+(1-\lambda) W_{2}^{\times n} .
$$

Note that $\boldsymbol{W}=\left\{W^{n}\right\}_{n=1}^{\infty} \in \mathcal{W}_{a}(E)$ since

$$
\begin{aligned}
e_{n}\left(P_{X}^{n}, W^{n}\right) & =\sum_{x^{n}, y^{n}} P_{X}^{n}\left(x^{n}\right) W^{n}\left(y^{n} \mid x^{n}\right) e_{n}\left(x^{n}, y^{n}\right) \\
& =\lambda e_{n}\left(P_{X}^{n}, W_{1}^{\times n}\right)+(1-\lambda) e_{n}\left(P_{X}^{n}, W_{2}^{\times n}\right) \\
& \leq \lambda E_{1}+(1-\lambda) E_{2} \\
& \leq E .
\end{aligned}
$$

Hence, by the definition of the achievability of $R$, for arbitrary small $\varepsilon>0$ and sufficiently large $n$, there exists a code $\left(\varphi_{n}, \psi_{n}\right)$ such that

$$
\frac{1}{n} \log \left|\mathcal{M}_{n}\right| \leq R+\varepsilon
$$

and

$$
\sum_{x^{n}, y^{n}} P_{X}^{n}\left(x^{n}\right) W^{n}\left(y^{n} \mid x^{n}\right) d_{n}\left(x^{n}, \psi_{n}\left(\varphi_{n}\left(x^{n}\right), y^{n}\right)\right) \leq D+\varepsilon .
$$

Note that (34) can be also written as

$$
\begin{aligned}
D+\varepsilon \geq \lambda & \sum_{x^{n}, y^{n}} P_{X}^{n}\left(x^{n}\right) W_{1}^{\times n}\left(y^{n} \mid x^{n}\right) d_{n}\left(x^{n}, \psi_{n}\left(\varphi_{n}\left(x^{n}\right), y^{n}\right)\right) \\
& +(1-\lambda) \sum_{x^{n}, y^{n}} P_{X}^{n}\left(x^{n}\right) W_{2}^{\times n}\left(y^{n} \mid x^{n}\right) d_{n}\left(x^{n}, \psi_{n}\left(\varphi_{n}\left(x^{n}\right), y^{n}\right)\right) .
\end{aligned}
$$

On the other hand, by using $\left(\varphi_{n}, \psi_{n}\right)$, we can construct a HB code $\left(\varphi_{n}^{H B}, \psi_{n}^{H B 1}, \psi_{n}^{H B 2}\right)$ as

$$
\begin{aligned}
\varphi_{n}^{H B}\left(x^{n}\right) & =\varphi_{n}\left(x^{n}\right) \quad x^{n} \in \mathcal{X}^{n}, \\
\psi_{n}^{H B 1}\left(m, y_{1}^{n}\right) & =\psi_{n}\left(m, y_{1}^{n}\right) \quad m \in \mathcal{M}_{n}, y_{1}^{n} \in \mathcal{Y}^{n}, \\
\psi_{n}^{H B 2}\left(m, y_{2}^{n}\right) & =\psi_{n}\left(m, y_{2}^{n}\right) \quad m \in \mathcal{M}_{n}, y_{2}^{n} \in \mathcal{Y}^{n} .
\end{aligned}
$$

Then, let $\left(D_{1}, D_{2}\right)$ be the pair of average distortion occurred by $\left(\varphi_{n}^{H B}, \psi_{n}^{H B 1}, \psi_{n}^{H B 2}\right)$, i.e.,

$$
D_{j}:=\sum_{x^{n}, y^{n}} P_{X}^{n}\left(x^{n}\right) W_{j}^{\times n}\left(y_{j}^{n} \mid x^{n}\right) d_{n}\left(x^{n}, \psi_{n}^{H B j}\left(\varphi_{n}^{H B}\left(x^{n}\right), y_{j}^{n}\right)\right) \quad j=1,2 .
$$

By the definition of $R_{H B}\left(D_{1}, D_{2} \mid W_{1}, W_{2}\right)$ and the construction of the code, we have

$$
R+\varepsilon \geq R_{H B}\left(D_{1}, D_{2} \mid W_{1}, W_{2}\right) .
$$

Further, 36) and (37) indicate

$$
D+\varepsilon \geq \lambda D_{1}+(1-\lambda) D_{2}
$$

Since we can choose $\varepsilon$ arbitrary small, we have $R \geq R_{H B}\left(D_{1}, D_{2} \mid W_{1}, W_{2}\right)$ and $\lambda D_{1}+(1-\lambda) D_{2} \leq D$. 


\section{B. Proof of Direct Part}

As the direct part proof of Theorem 6, we prove (18) in three steps. First, we construct a code for i.i.d. channel. Then, it is used to permutation invariant channels by using random permutation. Then, the size of the randomness is reduced by the de-randomization technique.

1) Code for i.i.d. channel: The goal of this section is to show the following lemma.

Lemma 23: For arbitrarily fixed $V \in \mathcal{V}(E, D)$ and $\delta>0$, there exists $\mu>0$ and a code $\varphi_{n}^{\prime}: \mathcal{X}^{n} \rightarrow \mathcal{U}^{n}$ such that

$$
\frac{1}{n} \log \left|\varphi_{n}^{\prime}\right| \leq I\left(P_{X}, V\right)+2 \delta
$$

and

$$
\operatorname{Pr}\left\{\left(\hat{U}^{n}, X^{n}, Y^{n}\right) \notin T_{P_{X} V \bar{W}, \delta}\right\} \leq 2^{-\mu n}
$$

for every $\bar{W} \in \mathcal{P}_{n}(\mathcal{Y} \mid \mathcal{X})$ provided that $n$ is sufficiently large, where $\left|\varphi_{n}^{\prime}\right|$ is the cardinality of the image of $\varphi_{n}^{\prime}, \hat{U}^{n}=\varphi_{n}^{\prime}\left(X^{n}\right)$ and $T_{P_{X} V \bar{W}, \delta}$ is the set of $P_{U X Y}$-typical sequences with respect to $P_{U X Y}(u, x, y)=$ $P_{X}(x) V(u \mid x) \bar{W}(y \mid x)$.

Proof: We construct a code in a similar manner as Section IV-B1. We use two kinds of bin codings $f_{n}: \mathcal{U}^{n} \rightarrow$ $\mathcal{S}_{n}$ and $g_{n}: \mathcal{U}^{n} \rightarrow \mathcal{L}_{n}$. We set

$$
\begin{aligned}
& R_{f}=H(U \mid X)-\delta, \\
& R_{g}=I\left(P_{X}, V\right)+2 \delta .
\end{aligned}
$$

Let $\left|\mathcal{S}_{n}\right|=\left\lfloor 2^{n R_{f}}\right\rfloor$ and $\left|\mathcal{L}_{n}\right|=\left\lceil 2^{n R_{g}}\right\rceil$.

Since

$$
R_{f}+R_{g}=H(U)+\delta
$$

there exists a decoder $\kappa_{n}: \mathcal{S}_{n} \times \mathcal{L}_{n} \rightarrow \mathcal{U}^{n}$ and $\mu_{1}>0$ such that

$$
\mathbb{E}_{F_{n} G_{n}}\left[P_{e r r}\left(F_{n}, G_{n}\right)\right] \leq 2^{-\mu_{1} n}
$$

for sufficiently large $n$, where $P_{\text {err }}\left(F_{n}, G_{n}\right)$ is the error probability of the source coding when the bin codings $\left(F_{n}, G_{n}\right)$ are used. Furthermore, since $R_{f}=H(U \mid X)-\delta, S_{n}=F_{n}\left(U^{n}\right)$ is close to the uniform random variable that is independent of $X^{n}$ (Lemma 30).

We construct a code as follows. Let

$$
P_{\hat{U}^{n} \mid S_{n} L_{n}}\left(u^{n} \mid s_{n}, \ell_{n}\right)=\mathbf{1}\left[u^{n}=\kappa_{n}\left(s_{n}, \ell_{n}\right)\right]
$$

be the distribution describing the decoder. Let

$$
\begin{aligned}
& P_{S_{n} L_{n} U^{n} X^{n} Y^{n} \hat{U}^{n}}\left(s_{n}, \ell_{n}, u^{n}, x^{n}, y^{n}, \hat{u}^{n}\right) \\
& \quad=P_{S_{n} X^{n}}\left(s_{n}, x^{n}\right) P_{U^{n} \mid S_{n} X^{n}}\left(u^{n} \mid s_{n}, x^{n}\right) P_{L_{n} \mid U^{n}}\left(\ell_{n} \mid u^{n}\right) P_{Y^{n} \mid X^{n}}\left(y^{n} \mid x^{n}\right) P_{\hat{U}^{n} \mid S_{n} L_{n}}\left(\hat{u}^{n} \mid s_{n}, \ell_{n}\right)
\end{aligned}
$$


and

$$
\begin{aligned}
& \hat{P}_{\bar{S}_{n} L_{n} U^{n} X^{n} Y^{n} \hat{U}^{n}}\left(s_{n}, \ell_{n}, u^{n}, x^{n}, y^{n}, \hat{u}^{n}\right) \\
& \quad=P_{\bar{S}_{n}}\left(s_{n}\right) P_{X^{n}}\left(x^{n}\right) P_{U^{n} \mid S_{n} X^{n}}\left(u^{n} \mid s_{n}, x^{n}\right) P_{L_{n} \mid U^{n}}\left(\ell_{n} \mid u^{n}\right) P_{Y^{n} \mid X^{n}}\left(y^{n} \mid x^{n}\right) P_{\hat{U}^{n} \mid S_{n} L_{n}}\left(\hat{u}^{n} \mid s_{n}, \ell_{n}\right) .
\end{aligned}
$$

Note that $P_{U^{n} \mid S_{n} X^{n}}$ is a randomized quantizer. From Lemma 30 and the fact that the variational distance does not increase by data processing and marginalization, we have

$$
\mathbb{E}_{F_{n} G_{n}}\left[\left\|\hat{P}_{\bar{S}_{n} U^{n} X^{n} Y^{n} \hat{U}^{n}}-P_{S_{n} U^{n} X^{n} Y^{n} \hat{U}^{n}}\right\|\right] \leq 2^{-\mu_{2} n}
$$

for some $\mu_{2}>0$. By Lemma 29 and 39 , we have

$$
\begin{aligned}
& \mathbb{E}_{F_{n} G_{n}}\left[P_{S_{n} U^{n} X^{n} Y^{n} \hat{U}^{n}}\left(\left\{\left(\hat{u}^{n}, x^{n}, y^{n}\right) \notin T_{P_{X} V W, \delta}\right\}\right)\right] \\
& \leq \mathbb{E}_{F_{n} G_{n}}\left[P_{S_{n} U^{n} X^{n} Y^{n} \hat{U}^{n}}\left(\left\{\left(u^{n}, x^{n}, y^{n}\right) \notin T_{P_{X} V W, \delta} \text { or } u^{n} \neq \hat{u}^{n}\right\}\right)\right] \\
& \leq 2^{-\mu_{3} n}
\end{aligned}
$$

for some $\mu_{3}>0$. Since

$$
\begin{aligned}
& \hat{P}_{\bar{S}_{n} U^{n} X^{n} Y^{n} \hat{U}^{n}}(\mathcal{A})-P_{S_{n} U^{n} X^{n} Y^{n} \hat{U}^{n}}(\mathcal{A}) \\
& \quad \leq\left\|\hat{P}_{\bar{S}_{n} U^{n} X^{n} Y^{n} \hat{U}^{n}}-P_{S_{n} U^{n} X^{n} Y^{n} \hat{U}^{n}}\right\|
\end{aligned}
$$

for any set $\mathcal{A}$, we have

$$
\mathbb{E}_{F_{n} G_{n}}\left[\hat{P}_{\bar{S}_{n} U^{n} X^{n} Y^{n} \hat{U}^{n}}\left(\left\{\left(\hat{u}^{n}, x^{n}, y^{n}\right) \notin T_{P_{X} V W, \delta}\right\}\right)\right] \leq 2^{-\mu_{4} n}
$$

for some $\mu_{4}>0$. Since the cardinality of $\mathcal{P}_{n}(\mathcal{Y} \mid \mathcal{X})$ is bounded by $(n+1)^{|\mathcal{X}||\mathcal{Y}|}$, there exists one realization $\left(f_{n}, g_{n}, s_{n}\right)$ of $\left(F_{n}, G_{n}, S_{n}\right)$ satisfying

$$
\hat{P}_{U^{n} X^{n} Y^{n} \hat{U}^{n} \mid \bar{S}_{n}}\left(\left\{\left(\hat{u}^{n}, x^{n}, y^{n}\right) \notin T_{P_{X} V W, \delta}\right\} \mid s_{n}\right) \leq(n+1)^{|\mathcal{X}||\mathcal{Y}|} 2^{-\mu_{4} n} .
$$

Furthermore, let $K_{n}$ be a random variable that simulate the randomized quantizer $P_{U^{n} \mid S_{n} X^{n}}$. Then, we can also eliminate this randomness in a similar manner. Let $\tau_{n}: \mathcal{S}_{n} \times \mathcal{X}^{n} \rightarrow \mathcal{U}^{n}$ be the resulting deterministic quantizer. Then, we set $\varphi_{n}^{\prime}\left(x^{n}\right)=\kappa_{n}\left(s_{n}, g_{n}\left(\tau_{n}\left(s_{n}, x^{n}\right)\right)\right)$. The image size of $\varphi_{n}^{\prime}$ obviously satisfies 38). Thus, by taking $n$ sufficiently large, we have the assertion of the lemma.

2) Code for Permutation Invariant Channel:

Lemma 24: For $\bar{W} \in \overline{\mathcal{W}}_{n}\left(T_{X, \delta}\right)$, let $\mathcal{A}_{n}(\bar{W}) \subset \mathcal{X}^{n} \times \mathcal{Y}^{n}$. Suppose that

$$
P_{X}^{n} \cdot \bar{W}^{\times n}\left(\mathcal{A}_{n}(\bar{W})^{c}\right) \leq \bar{\varepsilon}
$$

for every $\bar{W} \in \overline{\mathcal{W}}_{n}\left(T_{X, \delta}\right)$. Then, for any conditional type $\bar{W} \in \overline{\mathcal{W}}_{n}\left(T_{X, \delta}\right)$, we have

$$
\begin{aligned}
& \sum_{x^{n} \in T_{X, \delta}} P_{X}^{n}\left(x^{n}\right) \mathbf{1}\left[\bar{W} \in \mathcal{P}_{n}\left(\mathcal{Y} \mid P_{x^{n}}\right)\right] \\
& \quad \times \sum_{y^{n} \in T_{\bar{W}}\left(x^{n}\right)} \frac{1}{\left|T_{\bar{W}}\left(x^{n}\right)\right|} \mathbf{1}\left[\left(x^{n}, y^{n}\right) \in \mathcal{A}_{n}(\bar{W})^{c}\right] \\
& \leq\left.(n+1)^{|\mathcal{X}||\mathcal{Y}|}\right|_{\bar{\varepsilon} .}
\end{aligned}
$$


Proof: We prove this lemma in a similar manner as Lemma 19. For any $\bar{W} \in \mathcal{P}_{n}\left(\mathcal{Y} \mid P_{x^{n}}\right)$, note that (30) holds. Then, for any $\bar{W} \in \overline{\mathcal{W}}_{n}\left(T_{X, \delta}\right)$, we have

$$
\begin{aligned}
\bar{\varepsilon} & \geq P_{X}^{n} \cdot \bar{W}^{\times n}\left(\mathcal{A}_{n}(\bar{W})^{c}\right) \\
& \geq \sum_{x^{n} \in T_{X, \delta}} P_{X}^{n}\left(x^{n}\right) \mathbf{1}\left[\bar{W} \in \mathcal{P}_{n}\left(\mathcal{Y} \mid P_{x^{n}}\right)\right] \sum_{y^{n} \in T_{\bar{W}}\left(x^{n}\right)} \bar{W}^{\times n}\left(T_{\bar{W}}\left(x^{n}\right) \mid x^{n}\right) \frac{1}{\left|T_{\bar{W}}\left(x^{n}\right)\right|} \mathbf{1}\left[\left(x^{n}, y^{n}\right) \in \mathcal{A}_{n}(\bar{W})^{c}\right] \\
& \geq \frac{1}{(n+1)^{|\mathcal{X}||\mathcal{Y}|}} \sum_{x^{n} \in T_{X, \delta}} P_{X}^{n}\left(x^{n}\right) \mathbf{1}\left[\bar{W} \in \mathcal{P}_{n}\left(\mathcal{Y} \mid P_{x^{n}}\right)\right] \sum_{y^{n} \in T_{\bar{W}}\left(x^{n}\right)} \frac{1}{\left|T_{\bar{W}}\left(x^{n}\right)\right|} \mathbf{1}\left[\left(x^{n}, y^{n}\right) \in \mathcal{A}_{n}(\bar{W})^{c}\right],
\end{aligned}
$$

which implies the statement of the lemma.

Lemma 25: For a given $V \in \mathcal{V}\left(E+2 \delta e_{\max }, D\right)$, suppose that there exists $\varphi_{n}^{\prime}: \mathcal{X}^{n} \rightarrow \mathcal{U}^{n}$ such that

$$
\operatorname{Pr}\left\{\left(\hat{U}^{n}, X^{n}, Y^{n}\right) \notin T_{P_{X} V \bar{W}, \delta}\right\} \leq \bar{\varepsilon}
$$

for every $\bar{W} \in \mathcal{P}_{n}(\mathcal{Y} \mid \mathcal{X})$, where $\hat{U}^{n}=\varphi_{n}^{\prime}\left(X^{n}\right)$ and $T_{P_{X} V \bar{W}, \delta}$ is the set of all $P_{U X Y}$-typical set for $P_{U X Y}(u, x, y)=$ $P_{X}(x) V(u \mid x) \bar{W}(y \mid x)$. Then, we have

$$
\begin{aligned}
\mathbb{E} & {\left[d_{n}\left(X^{n}, \hat{U}^{n}\left(Y^{n}\right)\right)\right] } \\
& \leq\left\{P_{X}^{n}\left(T_{X, \delta}^{c}\right)+(n+1)^{2|\mathcal{X}||\mathcal{Y}|} \bar{\varepsilon}+\delta\right\} d_{\max }+D
\end{aligned}
$$

for every permutation invariant (not necessarily i.i.d.) $W^{n}$ such that

$$
\mathbb{E}\left[e_{n}\left(X^{n}, Y^{n}\right)\right] \leq E
$$

provided that $n$ is sufficiently large.

Proof: From [52), we first note that

$$
d_{n}\left(x^{n}, u^{n}\left(y^{n}\right)\right) \leq d(V, \bar{W})+\delta d_{\max }
$$

for $\left(u^{n}, x^{n}, y^{n}\right) \in T_{P_{X} V \bar{W}, \delta}$. Then, by using Lemma 24 for

$$
\mathcal{A}_{n}(\bar{W})=\left\{\left(x^{n}, y^{n}\right):\left(\varphi_{n}^{\prime}\left(x^{n}\right), x^{n}, y^{n}\right) \in T_{P_{X} V \bar{W}, \delta}\right\},
$$

we have

$$
\begin{aligned}
\mathbb{E}[ & \left.d_{n}\left(X^{n}, \hat{U}^{n}\left(Y^{n}\right)\right)\right] \\
\leq & P_{X}^{n}\left(T_{X, \delta}^{c}\right) d_{\max } \\
& +\sum_{\bar{W} \in \overline{\mathcal{W}}_{n}\left(T_{X, \delta}\right)} \sum_{x^{n} \in T_{X, \delta}} P_{X}^{n}\left(x^{n}\right) \mathbf{1}\left[\bar{W} \in \mathcal{P}_{n}\left(\mathcal{Y} \mid P_{x^{n}}\right)\right] \\
& \sum_{y^{n} \in T_{\bar{W}}\left(x^{n}\right)} W^{n}\left(T_{\bar{W}}\left(x^{n}\right) \mid x^{n}\right) \frac{1}{\left|T_{\bar{W}}\left(x^{n}\right)\right|} \mathbf{1}\left[\left(x^{n}, y^{n}\right) \in \mathcal{A}_{n}^{c}(\bar{W})\right] d_{\max } \\
& +\sum_{\bar{W} \in \overline{\mathcal{W}}_{n}\left(T_{X, \delta}\right)} \sum_{x^{n} \in T_{X, \delta}} P_{X}^{n}\left(x^{n}\right) \mathbf{1}\left[\bar{W} \in \mathcal{P}_{n}\left(\mathcal{Y} \mid P_{x^{n}}\right)\right] \\
& \sum_{y^{n} \in T_{\bar{W}}\left(x^{n}\right)} W^{n}\left(T_{\bar{W}}\left(x^{n}\right) \mid x^{n}\right) \frac{1}{\left|T_{\bar{W}}\left(x^{n}\right)\right|} \mathbf{1}\left[\left(x^{n}, y^{n}\right) \in \mathcal{A}_{n}(\bar{W})\right]\left\{d(V, \bar{W})+\delta d_{\max }\right\}
\end{aligned}
$$




$$
\begin{aligned}
\leq & \left\{P_{X}^{n}\left(T_{X, \delta}^{c}\right)+(n+1)^{2|\mathcal{X}||\mathcal{Y}|} \bar{\varepsilon}+\delta\right\} d_{\max } \\
& +\sum_{x^{n} \in T_{X, \delta}} \sum_{\bar{W} \in \mathcal{P}_{n}\left(\mathcal{Y} \mid P_{x^{n}}\right)} P_{X}^{n}\left(x^{n}\right) W^{n}\left(T_{\bar{W}}\left(x^{n}\right) \mid x^{n}\right) d(V, \bar{W}),
\end{aligned}
$$

where we used Lemma 24 to upper bound the second term in the second inequality. Now, we rewrite the last term as

$$
\begin{aligned}
& \sum_{x^{n} \in T_{X, \delta}} \sum_{\bar{W} \in \mathcal{P}_{n}\left(\mathcal{Y} \mid P_{x}^{n}\right)} P_{X}^{n}\left(x^{n}\right) W^{n}\left(T_{\bar{W}}\left(x^{n}\right) \mid x^{n}\right) d(V, \bar{W}) \\
& =P_{X}^{n}\left(T_{X, \delta}\right) d\left(V, W_{\text {mix }}\right),
\end{aligned}
$$

where $W_{\text {mix }} \in \mathcal{P}(\mathcal{Y} \mid \mathcal{X})$ is a channel defined by

$$
W_{m i x}(y \mid x)=\sum_{x^{n} \in T_{X, \delta}} \tilde{P}_{X}^{n}\left(x^{n}\right) \sum_{\bar{W} \in \mathcal{P}_{n}\left(\mathcal{Y} \mid P_{x}^{n}\right)} W^{n}\left(T_{\bar{W}}\left(x^{n}\right) \mid x^{n}\right) \bar{W}(y \mid x)
$$

for

$$
\tilde{P}_{X}^{n}\left(x^{n}\right)=\frac{P_{X}^{n}\left(x^{n}\right)}{P_{X}^{n}\left(T_{X, \delta}\right)}
$$

From (49) and 50), we have

$$
e_{n}\left(x^{n}, y^{n}\right) \geq e\left(P_{X}, \bar{W}\right)-\delta e_{\max }
$$

for $x^{n} \in T_{X, \delta}$ and $y^{n} \in T_{\bar{W}}\left(x^{n}\right)$. Thus, we have

$$
\begin{aligned}
E & \geq \mathbb{E}\left[e_{n}\left(X^{n}, Y^{n}\right)\right] \\
& =\sum_{x^{n}, y^{n}} P_{X}^{n}\left(x^{n}\right) W^{n}\left(y^{n} \mid x^{n}\right) e_{n}\left(x^{n}, y^{n}\right) \\
& \geq \sum_{x^{n} \in T_{X, \delta}} P_{X}^{n}\left(x^{n}\right) \sum_{\bar{W} \in \mathcal{P}_{n}\left(\mathcal{Y} \mid P_{x} n\right)} W^{n}\left(T_{\bar{W}}\left(x^{n}\right) \mid x^{n}\right) e\left(P_{x^{n}}, \bar{W}\right) \\
& \geq \sum_{x^{n} T_{X, \delta}} P_{X}^{n}\left(x^{n}\right) \sum_{\bar{W} \in \mathcal{P}_{n}\left(\mathcal{Y} \mid P_{x^{n}}\right)} W^{n}\left(T_{\bar{W}}\left(x^{n}\right) \mid x^{n}\right)\left\{e\left(P_{X}, \bar{W}\right)-\delta e_{\max }\right\} \\
& =P_{X}^{n}\left(T_{X, \delta}\right)\left\{e\left(P_{X}, W_{\text {mix }}\right)-\delta e_{\max }\right\} .
\end{aligned}
$$

Thus, we have $W_{\text {mix }} \in \mathcal{W}_{1}\left(E+2 \delta e_{\max }\right)$ provided that $n$ is sufficiently large. Since $V \in \mathcal{V}\left(E+2 \delta e_{\max }, D\right)$, we have $d\left(V, W_{\text {mix }}\right) \leq D$. This completes the proof.

By combining Lemma 23 and Lemma 25, we have the following.

Lemma 26: For any $V \in \mathcal{V}\left(E+2 \delta e_{\max }, D\right), \delta>0$, and $\varepsilon>0$, there exists $\varphi_{n}^{\prime}: \mathcal{X}^{n} \rightarrow \mathcal{U}^{n}$ such that

$$
\frac{1}{n} \log \left|\varphi_{n}^{\prime}\right| \leq I\left(P_{X}, V\right)+\delta
$$

and

$$
\mathbb{E}_{\pi_{n}}\left[\mathbb{E}\left[d_{n}\left(\pi_{n}\left(X^{n}\right), \hat{U}^{n}\left(\pi_{n}\left(Y^{n}\right)\right)\right)\right]\right] \leq D+\varepsilon
$$

for every $\mathbf{W} \in \mathcal{W}_{m}(E)$ provided that $n$ is sufficiently large, where $\hat{U}^{n}=\varphi_{n}^{\prime}\left(\pi_{n}\left(X^{n}\right)\right)$. 
3) De-Randomization: Now we reduce the size of random permutation by using the de-randomization technique.

Lemma 27: Suppose that $\varphi_{n}^{\prime}$ satisfies (40). Then, for arbitrary $\delta_{2}, \gamma>0$, there exists $m_{n}=2^{\delta_{2} n}$ permutations $\left\{\pi_{n}^{(1)}, \ldots, \pi_{n}^{\left(m_{n}\right)}\right\}$ such that

$$
\frac{1}{m_{n}} \sum_{i=1}^{m_{n}} \mathbb{E}\left[d_{n}\left(\pi_{n}\left(X^{n}\right), \hat{U}^{n}\left(\pi_{n}\left(Y^{n}\right)\right)\right)\right] \leq D+\varepsilon+\gamma
$$

provided that $n$ is sufficiently large.

Proof: For a permutation $\pi_{n}$ and $\left(x^{n}, y^{n}\right) \in \mathcal{X}^{n} \times \mathcal{Y}^{n}$, we denote

$$
J\left(\pi_{n}, x^{n}, y^{n}\right)=d_{n}\left(\pi_{n}\left(x^{n}\right), \hat{u}^{n}\left(\pi_{n}\left(y^{n}\right)\right)\right),
$$

where $\hat{u}^{n}=\varphi_{n}^{\prime}\left(\pi_{n}\left(x^{n}\right)\right)$. Let $\pi_{n}^{(1)}, \ldots, \pi_{n}^{\left(m_{n}\right)}$ be randomly generated permutations, and let $\bar{J}\left(x^{n}, y^{n}\right)=\mathbb{E}_{\pi_{n}}\left[J\left(\pi_{n}, x^{n}, y^{n}\right)\right]$. Then, by using Lemma 31 for $A_{i}=J\left(\pi_{n}^{(i)}, x^{n}, y^{n}\right), b=d_{\max }$, and $\alpha=\frac{\gamma}{2 d_{\max }^{2}}$, we have

$$
\begin{aligned}
& \operatorname{Pr}\left\{\frac{1}{m_{n}} \sum_{i=1}^{m_{n}} J\left(\pi_{n}^{(i)}, x^{n}, y^{n}\right) \geq \bar{J}\left(x^{n}, y^{n}\right)+\gamma\right\} \\
& \quad \leq \exp \left\{-\left(\gamma^{2} / 4 d_{\max }^{2}\right) m_{n}\right\} .
\end{aligned}
$$

Furthermore, by using the union bound, we have

$$
\begin{aligned}
& \operatorname{Pr}\left\{\exists\left(x^{n}, y^{n}\right) \frac{1}{m_{n}} \sum_{i=1}^{m_{n}} J\left(\pi_{n}^{(i)}, x^{n}, y^{n}\right) \geq \bar{J}\left(x^{n}, y^{n}\right)+\gamma\right\} \\
& \quad \leq\left|\mathcal{X}^{n}\right|\left|\mathcal{Y}^{n}\right| \exp \left\{-\left(\gamma^{2} / 4 d_{\max }^{2}\right) m_{n}\right\} .
\end{aligned}
$$

Since $\exp \left\{-\left(\gamma^{2} / 4 d_{\max }^{2}\right) m_{n}\right\}$ converges to 0 doubly exponentially, the righthand side of 410 is strictly smaller than 1 if $n$ is sufficiently large, which implies that there exists one realization of $\pi_{n}^{(1)}, \ldots, \pi_{n}^{\left(m_{n}\right)}$ such that

$$
\frac{1}{m_{n}} \sum_{i=1}^{m_{n}} J\left(\pi_{n}^{(i)}, x^{n}, y^{n}\right) \leq \bar{J}\left(x^{n}, y^{n}\right)+\gamma
$$

for every $\left(x^{n}, y^{n}\right)$. Finally, by taking the average over both sides of (42) with respect to $\left(X^{n}, Y^{n}\right)$, we have the assertion of the lemma.

Finally, by combining Lemma 26 and Lemma 27 and by taking the constants to be sufficiently small and $n$ to be sufficiently large, we can show that the righthand side of (18) is achievable.

\section{CONCLUSION}

In this paper, we introduced the novel rate-distortion functions for the Wyner-Ziv problem, which are defined as the minimum rates required for the universal coding for the distortion constrained general channel classes. Then, we derived the upper bounds and lower bounds on the rate-distortion functions. The complete solution for the rate-distortion functions is remained open. Parts of difficulties are related to the Heegard-Berger problem, which is also a long-standing open problem. 


\section{APPENDIX}

\section{A. Proof of Proposition 2}

From the definition, for any $\nu>0$ there exists an average-achievable rate $R$ such that $R \leq R_{a}(D \mid E)+\nu$. For any $\epsilon>0$ and $\boldsymbol{W} \in \mathcal{W}_{m}(E-\epsilon)$, we have

$$
\begin{aligned}
\mathbb{E}\left[e_{n}\left(P_{X^{n}}, W^{n}\right)\right] & \leq E-\epsilon+e_{\max } \operatorname{Pr}\left\{e_{n}\left(X^{n}, Y^{n}\right)>E\right\} \\
& \leq E
\end{aligned}
$$

provided that $n \geq n_{0}\left(\epsilon / e_{\max }\right)$.

Let $\tilde{\boldsymbol{W}}=\left\{\tilde{W}^{n}\right\}_{n=1}^{\infty}$ be a sequence of channels such that $\tilde{W}^{n}=W^{n}$ for $n \geq n_{0}\left(\epsilon / e_{\max }\right)$ and $\tilde{W}^{n}$ for $1 \leq$ $n<n_{0}\left(\epsilon / e_{\max }\right)$ are chosen appropriately so that $\mathbb{E}\left[e_{n}\left(P_{X^{n}}, \tilde{W}^{n}\right)\right] \leq E$. Then from (44), we have $\tilde{\boldsymbol{W}} \in \mathcal{W}_{a}(E)$. Then, since $R$ is average achievable, for any $\varepsilon>0$ there exists a code such that (4) and (5) are satisfied for $\tilde{W}^{n}$ and $n \geq n_{1}(\varepsilon)$. This also implies that the code also satisfies (4) and (5) for $W^{n}$ and $n \geq \max \left[n_{0}\left(\epsilon / e_{\max }\right), n_{1}(\varepsilon)\right]$. Since $\boldsymbol{W} \in \mathcal{W}_{m}(E-\epsilon)$ is arbitrary, $R$ is also maximum-achievable. Since $\nu>0$ is arbitrary, we have

$$
R_{a}(D \mid E) \geq R_{m}(D \mid E-\epsilon)
$$

Thus, by taking the limit $\epsilon \rightarrow 0$, we have the assertion of the proposition.

\section{B. Continuity of $R_{W Z}(D \mid W)$}

Lemma 28: For $D>0, R_{W Z}(D \mid W)$ is a continuous function with respect to $W$.

Proof: For two channels $W_{1}, W_{2}$, we consider the distance given by

$$
\Delta\left(W_{1}, W_{2}\right):=\sum_{x, y}\left|P_{X}(x) W_{1}(y \mid x)-P_{X}(x) W_{2}(y \mid x)\right| .
$$

Since the Euclidian distance $\left\|W_{1}-W_{2}\right\|_{2}$ converging to 0 implies $\Delta\left(W_{1}, W_{2}\right)$ converging to 0 , it suffice to show the continuity of $R_{W Z}(D \mid W)$ with respect to the topology given by $\Delta(\cdot, \cdot)$.

By a slight abuse of notation, we also introduce

$$
\Delta\left(V_{1}, V_{2}\right):=\sum_{u, x}\left|P_{X}(x) V_{1}(u \mid x)-P_{X}(x) V_{2}(u \mid x)\right|
$$

for two test channel $V_{1}, V_{2}$. From the definition of the variational distance, we can find that

$$
\Delta\left(W_{1}, W_{2}\right)=\left\|P_{X} V W_{1}-P_{X} V W_{2}\right\|
$$

for any fixed test channel $V$ and

$$
\Delta\left(V_{1}, V_{2}\right)=\left\|P_{X} V_{1} W-P_{X} V_{2} W\right\|
$$

for any fixed channel $W$, where $P_{X} V W$ is the joint distribution given by $P_{X}(x) V(u \mid x) W(y \mid x)$. Furthermore, from Fannes' inequality [32, Lemma 2.7], there exists a function $\nu(\delta)$ such that $\nu(\delta) \rightarrow 0$ as $\delta \rightarrow 0$ and

$$
\left|\phi\left(V, W_{1}\right)-\phi\left(V, W_{2}\right)\right| \leq \nu\left(\Delta\left(W_{1}, W_{2}\right)\right)
$$


for fixed $V$ and

$$
\left|\phi\left(V_{1}, W\right)-\phi\left(V_{2}, W\right)\right| \leq \nu\left(\Delta\left(V_{1}, V_{2}\right)\right)
$$

for fixed $W$.

For two channels $W_{1}, W_{2}$, let $\epsilon:=d_{\max } \Delta\left(W_{1}, W_{2}\right)$. Let $V_{i}^{*} \in \mathcal{V}\left(W_{i}, D+\epsilon\right)$ be a test channel such that

$$
\phi\left(V_{i}^{*}, W_{i}\right)=R_{W Z}\left(D+\epsilon \mid W_{i}\right) .
$$

Let $\tilde{V}_{i}$ be a test channel such that $\tilde{V}_{i} \in \mathcal{V}\left(W_{i}, 0\right)$. We set

$$
V_{i}^{\dagger}:=\frac{D}{D+\epsilon} V_{i}^{*}+\frac{\epsilon}{D+\epsilon} \tilde{V}_{i}
$$

Then, we have

$$
\begin{aligned}
\Delta\left(V_{i}^{*}, V_{i}^{\dagger}\right) & \leq \frac{\epsilon}{D+\epsilon}\left\|P_{X} V_{i}^{*}-P_{X} \tilde{V}_{i}\right\| \\
& \leq \frac{2 \epsilon}{D+\epsilon}
\end{aligned}
$$

and

$$
\begin{aligned}
d\left(V_{i}^{\dagger}, W_{i}\right) & =\frac{D}{D+\epsilon} d\left(V_{i}^{*}, W_{i}\right)+\frac{\epsilon}{D+\epsilon} d\left(\tilde{V}_{i}, W_{i}\right) \\
& \leq D
\end{aligned}
$$

Furthermore, let $V_{i}^{\ddagger} \in \mathcal{V}\left(W_{i}, D\right)$ be such that

$$
\phi\left(V_{i}^{\ddagger}, W_{i}\right)=R_{W Z}\left(D \mid W_{i}\right) .
$$

Note that $V_{1}^{\ddagger} \in \mathcal{V}\left(W_{2}, D+\epsilon\right)$ and $V_{2}^{\ddagger} \in \mathcal{V}\left(W_{1}, D+\epsilon\right)$.

By using above notations, we have

$$
\begin{aligned}
R_{W Z}\left(D \mid W_{1}\right) & =\phi\left(V_{1}^{\ddagger}, W_{1}\right) \\
& \stackrel{\text { (a) }}{\geq}\left(V_{1}^{\ddagger}, W_{2}\right)-\nu\left(\Delta\left(W_{1}, W_{2}\right)\right) \\
& \stackrel{\text { (b) }}{\geq}\left(V_{2}^{*}, W_{2}\right)-\nu\left(\Delta\left(W_{1}, W_{2}\right)\right) \\
& \stackrel{(\text { c) }}{\geq} \phi\left(V_{2}^{\dagger}, W_{2}\right)-\nu\left(\Delta\left(W_{1}, W_{2}\right)\right)-\nu(2 \epsilon /(D+\epsilon)) \\
& \stackrel{\text { (d) }}{\geq}\left(V_{2}^{\ddagger}, W_{2}\right)-\nu\left(\Delta\left(W_{1}, W_{2}\right)\right)-\nu(2 \epsilon /(D+\epsilon)) \\
& =R_{W Z}\left(D \mid W_{2}\right)-\nu\left(\Delta\left(W_{1}, W_{2}\right)\right)-\nu(2 \epsilon /(D+\epsilon)),
\end{aligned}
$$

where (a) follows from (45), (b) follows from $V_{1}^{\ddagger} \in \mathcal{V}\left(W_{2}, D+\epsilon\right)$ and the fact that $V_{2}^{*}$ minimizes $\phi\left(V, W_{2}\right)$ under $V \in \mathcal{V}\left(W_{2}, D+\epsilon\right)$, (c) follows from (48) and (46), and (d) follows from $V_{2}^{\dagger} \in \mathcal{V}\left(W_{2}, D\right)$ and the fact that $V_{2}^{\ddagger}$ minimizes $\phi\left(V, W_{2}\right)$ under $V \in \mathcal{V}\left(W_{2}, D\right)$. Similarly, we can prove the inequality in which $W_{1}$ and $W_{2}$ are interchanged. Thus we have

$$
\left|R_{W Z}\left(D \mid W_{1}\right)-R_{W Z}\left(D \mid W_{2}\right)\right| \leq \nu\left(\Delta\left(W_{1}, W_{2}\right)\right)+\nu(2 \epsilon /(D+\epsilon)) .
$$

Since $\epsilon \rightarrow 0$ as $\Delta\left(W_{1}, W_{2}\right) \rightarrow 0$, we have proved the continuity of $R_{W Z}(D \mid W)$. 


\section{Miscellaneous Facts on Types and Typicality}

In this section, we introduce some notations and known facts on the type method [32].

The type of a sequence $x^{n}$ and the joint type of $\left(x^{n}, y^{n}\right)$ are denoted by $P_{x^{n}}$ and $P_{x^{n}} y^{n}$ respectively. The set of all types and joint types are denoted by $\mathcal{P}_{n}(\mathcal{X})$ and $\mathcal{P}_{n}(\mathcal{X} \times \mathcal{Y})$. For type $P$, the set of all sequence such that $P_{x^{n}}=P$ is denoted by $T_{P}$. We use a similar notation for joint types. The set of all conditional types is denoted by $\mathcal{P}_{n}(\mathcal{Y} \mid \mathcal{X})$, and the set of $W$-shell for given $x^{n}$ is denoted by $T_{W}\left(x^{n}\right)$. For type $P \in \mathcal{P}_{n}(\mathcal{X})$, the set of all conditional types such that $T_{W}\left(x^{n}\right)$ is not empty is denoted by $\mathcal{P}_{n}(\mathcal{Y} \mid P)$. It is well known that

$$
\begin{aligned}
\left|\mathcal{P}_{n}(\mathcal{X})\right| & \leq(n+1)^{|\mathcal{X}|} \\
\left|\mathcal{P}_{n}(\mathcal{X} \times \mathcal{Y})\right| & \leq(n+1)^{|\mathcal{X}||\mathcal{Y}|} \\
\left|\mathcal{P}_{n}(\mathcal{Y} \mid \mathcal{X})\right| & \leq(n+1)^{|\mathcal{X}||\mathcal{Y}|},
\end{aligned}
$$

and these inequalities are extensively used in the paper.

For $P_{X} \in \mathcal{P}(\mathcal{X})$, a sequence $x^{n}$ is called $P_{X}$-typical sequence with constant $\delta$ if

$$
\left|P_{x^{n}}(a)-P_{X}(a)\right| \leq \delta \forall a \in \mathcal{X}
$$

and no $a \in \mathcal{X}$ with $P_{X}(a)=0$ occurs in $x^{n}$. The set of all typical sequence is denoted by $T_{X, \delta}$. The set of all types $P \in \mathcal{P}_{n}(\mathcal{X})$ such that $T_{P} \subset T_{X, \delta}$ is denoted by $\mathcal{P}_{X, \delta, n}$. For joint probability distribution, joint typical sequence and the set of all joint typical sequences are defined in a similar manner. It is well known that the set of all non-typical sequences occur with exponential small probability. Especially for our purpose, we need a bound such that the convergence is uniform with respect to $P_{X}$.

Lemma 29: For any $P_{X} \in \mathcal{P}(\mathcal{X})$, we have

$$
P_{X}^{n}\left(T_{X, \delta}^{c}\right) \leq 2|\mathcal{X}| 2^{-n \frac{2 \delta^{2}}{5 \ln 2}}
$$

Proof: For each $a \in \mathcal{X}$ such that $P_{X}(a)>0$, by noting that the variance of $\mathbf{1}\left[X_{i}=a\right]-P_{X}(a)$ is bounded by $\frac{1}{2}$ and $\left|\mathbf{1}\left[X_{i}=a\right]-P_{X}(a)\right| \leq 1$ with probability one, and by using the Bernstein inequality, we have

$$
\operatorname{Pr}\left\{\left|P_{X^{n}}(a)-P_{X}(a)\right| \geq \delta\right\} \leq 2 \cdot 2^{-n \frac{2 \delta^{2}}{5 \ln 2}}
$$

for any $0<\delta \leq 1$. Thus, by using the union bound with respect to $a \in \mathcal{X}$, we have the assertion.

Since the distortion is additive, the distortion between $x^{n}$ and $y^{n}$ only depends on their joint type, and thus we have

$$
e_{n}\left(x^{n}, y^{n}\right)=e(P, W)
$$

if $x^{n} \in T_{P}$ and $y^{n} \in T_{W}\left(x^{n}\right)$. From the definition of $\mathcal{P}_{X, \delta, n}$, we have

$$
\left|e(P, W)-e\left(P_{X}, W\right)\right| \leq \delta e_{\max }
$$

for any $P \in \mathcal{P}_{X, \delta, n}$ and $W \in \mathcal{P}(\mathcal{Y} \mid \mathcal{X})$. 
For $P \in \mathcal{P}_{n}(\mathcal{X})$, let

$$
\overline{\mathcal{W}}_{n}(P, E):=\mathcal{W}_{1}(P, E) \cap \mathcal{P}_{n}(\mathcal{Y} \mid P)
$$

Then, for $P \in \mathcal{P}_{X, \delta, n}$, 50) implies

$$
W \in \overline{\mathcal{W}}_{n}(P, E) \Longrightarrow W \in \mathcal{W}_{1}\left(E+\delta e_{\max }\right) .
$$

We also use the notation

$$
\begin{aligned}
\overline{\mathcal{W}}_{n}\left(T_{X, \delta}, E\right) & :=\bigcup_{P \in \mathcal{P}_{X, \delta, n}} \overline{\mathcal{W}}_{n}(P, E), \\
\overline{\mathcal{W}}_{n}\left(T_{X, \delta}\right) & :=\bigcup_{P \in \mathcal{P}_{X, \delta, n}} \mathcal{P}_{n}(\mathcal{Y} \mid P) .
\end{aligned}
$$

For $(V, W) \in \mathcal{P}(\mathcal{U} \mid \mathcal{X}) \times \mathcal{P}(\mathcal{Y} \mid \mathcal{X})$, let $P_{U X Y}(u, x, y)=P_{X}(x) V(y \mid x) W(y \mid x)$. For $\left(u^{n}, x^{n}, y^{n}\right) \in T_{U X Y, \delta}$, by the same reason as (49) and (50), we have

$$
\left|d_{n}\left(x^{n}, u^{n}\left(y^{n}\right)\right)-d(V, W)\right| \leq \delta d_{\max } .
$$

\section{Privacy Amplification Lemma}

Lemma 30: Let $F_{n}$ be the random binning from $\mathcal{U}^{n}$ to $\mathcal{S}_{n}$ such that $\left|\mathcal{S}_{n}\right|=\left\lfloor 2^{n R_{f}}\right\rfloor$, where $R_{f}=H(U \mid X)-\delta$. Then, there exists $\mu_{2}>0$ such that

$$
\mathbb{E}_{F_{n}}\left[\left\|P_{S_{n} X^{n}}-P_{\bar{S}_{n}} \times P_{X^{n}}\right\|\right] \leq 2^{-\mu_{2} n},
$$

where $P_{\bar{S}_{n}}$ is the uniform distribution on $\mathcal{S}_{n}$.

Proof: The lemma is a straightforward consequence of [33, (51)], which states that

$$
\mathbb{E}\left[\left\|P_{S_{n} X^{n}}-P_{\bar{S}_{n}} \times P_{X^{n}}\right\|\right] \leq 3\left|\mathcal{S}_{n}\right|^{\theta} 2^{n \tau\left(\theta \mid P_{U X}\right)}
$$

for $0 \leq \theta \leq \frac{1}{2}$, where

$$
\tau\left(\theta \mid P_{U X}\right)=\log \sum_{x} P_{X}(x)\left(\sum_{u} P_{U \mid X}(u \mid x)^{\frac{1}{1-\theta}}\right)^{1-\theta} .
$$

Since $\left.\frac{d \tau\left(\theta \mid P_{U X}\right)}{d \theta}\right|_{\theta=0}=-H(U \mid X)$, there exists $\theta_{0}>0$ such that

$$
\frac{\tau\left(\theta_{0} \mid P_{U X}\right)}{\theta_{0}} \leq-H(U \mid X)+\frac{\delta}{2}
$$

Thus, we have

$$
\begin{aligned}
\frac{\theta_{0}}{n} \log \left|\mathcal{S}_{n}\right|+\tau\left(\theta_{0} \mid P_{U X}\right) & \leq R_{f}-H(U \mid X)+\frac{\delta}{2} \\
& =-\frac{\delta}{2} .
\end{aligned}
$$

Combining (53) and (54), we have the assertion of the lemma. 


\section{E. Bernstein's Trick}

Lemma 31 ([28]): Let $A_{1}, \ldots, A_{m}$ be a sequence of discrete independent random variables that take values in $[-b, b]$. Then, for $0<\alpha \leq \min \left[1, \frac{b^{n}}{2} e^{-2 b}\right]$, we have

$$
\operatorname{Pr}\left\{\frac{1}{m} \sum_{i=1}^{m}\left(A_{i}-\mathbb{E}\left[A_{i}\right]\right) \geq \gamma\right\} \leq \exp \left\{\left(-\alpha \gamma+\alpha^{2} b^{2}\right) m\right\} .
$$

\section{REFERENCES}

[1] A. D. Wyner and J. Ziv, "The rate-distortion function for source coding with side information at the decoder," IEEE Trans. Inform. Theory, vol. 22, no. 1, pp. 1-10, January 1976.

[2] D. Slepian and J. K. Wolf, "Noiseless coding of correlated information sources," IEEE Trans. Inform. Theory, vol. 19, no. 4, pp. 471-480, July 1973.

[3] I. Csiszár and Körner, "Graph decomposition: A new key to coding theorems," IEEE Trans. Inform. Theory, vol. 27, no. 1, pp. 5-12, January 1981.

[4] I. Csiszár, "Linear codes for sources and source networks: Error exponents, universal coding," IEEE Trans. Inform. Theory, vol. 28, no. 4, pp. 585-592, July 1982.

[5] Y. Oohama and T. S. Han, "Universal coding for the Slepian-Wolf data compression system and the strong converse theorem," IEEE Trans. Inform. Theory, vol. 40, no. 6, pp. 1908-1919, November 1994.

[6] Y. Oohama, "Universal coding for correlated sources with linked encoders," IEEE Trans. Inform. Theory, vol. 42, no. 3, pp. 837-843, May 1996.

[7] A. Kimura, T. Uyematsu, S. Kuzuoka, and S. Watanabe, "Universal source coding over generalized complementary delivery networks," IEEE Trans. Inform. Theory, vol. 55, no. 3, pp. 1360-1373, March 2009.

[8] N. Merhav and J. Ziv, "On the Wyner-Ziv problem for individual sequences," IEEE Trans. Inform. Theory, vol. 52, no. 3, pp. 867-873, March 2006.

[9] S. Jalali, S. Verdu, and T. Weissman, "A universal scheme for Wyner-Ziv coding of discrete sources," IEEE Trans. Inform. Theory, vol. 56, no. 4, pp. 1737-1750, April 2010.

[10] A. Reani and N. Merhav, "Efficient on-line scheme for encoding individual sequences with side information at the decoder," IEEE Trans. Inform. Theory, vol. 57, no. 10, pp. 6860-6876, October 2011.

[11] S. Kuzuoka, A. Kimura, and T. Uyematsu, "Universal source coding for multiple decoders with side information," in IEEE International Symposium on Information Theory, 2010, pp. 1-5.

[12] C. Heegard and T. Berger, "Rate distortion when side information may be absent," IEEE Trans. Inform. Theory, vol. 31, no. 6, pp. 727-734, November 1985.

[13] Y. Steinberg and N. Merhav, "On successive refinement for the Wyner-Ziv problem," IEEE Trans. Inform. Theory, vol. 50, no. 8, pp. 1636-1654, August 2004.

[14] C. Tian and S. Diggavi, "On multistage successive refinement for Wyner-Ziv source coding with degraded side informations," IEEE Trans. Inform. Theory, vol. 53, no. 8, pp. 2946-2960, August 2007.

[15] _ _ "Side-information scalable source coding," IEEE Trans. Inform. Theory, vol. 54, no. 12, pp. 5591-508, December 2008.

[16] R. Timo, T. Chan, and A. Grant, "Rate distortion with side-information at many decoders," IEEE Trans. Inform. Theory, vol. 57, no. 8, pp. 5240-5257, August 2011.

[17] S. Watanabe, "The rate-distortion function for product of two sources with side-information at decoders," in Proc. IEEE Int. Symp. Inf. Theory 2011, Saint Petersburg, Russia, 2011, pp. 2862-2866, arXiv:1105.2864.

[18] W. H. R. Equitz and T. M. Cover, "Successive refinement of information," IEEE Trans. Inform. Theory, vol. 37, no. 2, pp. 269-275, March 1991.

[19] B. Rimoldi, "Successive refinement of information: Characterization of the achievable rates," IEEE Trans. Inform. Theory, vol. 40, no. 1, pp. 253-259, January 1994.

[20] P. Mouline and J. A. O'Sullivan, "Information-theoretic analysis of information hiding," IEEE Trans. Inform. Theory, vol. 49, no. 3, pp. 563-593, March 2003. 
[21] A. S. Cohen and A. Lapidoth, "The gaussian watermarking game," IEEE Trans. Inform. Theory, vol. 48, no. 6, pp. 1639-1667, June 2002.

[22] A. S.-Baruch and N. Merhav, "On the error exponent and capacity games of private watermarking systems," IEEE Trans. Inform. Theory, vol. 49, no. 3, pp. 537-562, March 2003.

[23] R. J. Barron, B. Chen, and G. W. Wornell, "The duality between information embedding and source coding with side information and some applications," IEEE Trans. Inform. Theory, vol. 49, no. 5, pp. 1159-1180, May 2003.

[24] T. M. Cover and M. Chiang, "Duality between channel capacity and rate distortion with two-sided state information," IEEE Trans. Inform. Theory, vol. 48, no. 6, pp. 1629-1638, June 2002.

[25] A. El Gamal and Y.-H. Kim, Network Information Theory. Cambridge, 2011.

[26] F. M. J. Willems, "Computation of the Wyner-Ziv rate distortion function," in Eindhoven Univ. Tech. Rep. 83-E-140, 1983.

[27] D. P. Bertsekas, A. Nedić, and A. E. Ozdaglar, Convex Analysis and Optimization. Athena Scientific, 2003.

[28] R. Ahlswede, "A method of coding and an application to arbitrary varying channel," J. Comb. Inform. Syst., vol. 5, no. 1, pp. 10-35, 1980.

[29] J. Ziv, "Fixed-rate encoding of individual sequences with side information," IEEE Trans. Inform. Theory, vol. 30, no. 2, pp. 348-352, March 1984.

[30] M. H. Yassaee, M. R. Aref, and A. Gohari, “Achievability proof via output statistics of random binning," 2012, arXiv:1203.0730.

[31] B. G. Kelly and A. B. Wagner, "Reliability in source coding with side information," IEEE Trans. Inform. Theory, vol. 58, no. 8, pp. 5086-5111, August 2012, arXiv:1109.0923.

[32] I. Csiszár and J. Körner, Information Theory, Coding Theorems for Discrete Memoryless Systems, 2nd ed. Cambridge University Press, 2011.

[33] M. Hayashi, “Tight exponential evaluation for information theoretical secrecy based on $l_{1}$ distance," arXiv:1010.1358. 